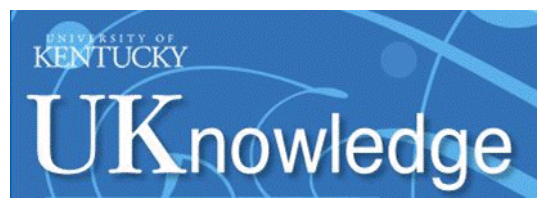

University of Kentucky

UKnowledge

Theses and Dissertations--Kinesiology and Health Promotion

2016

\title{
THE EFFECTS OF A HIP FLEXOR STRETCHING PROGRAM ON RUNNING KINEMATICS IN INDIVIDUALS WITH LIMITED PASSIVE HIP EXTENSION
}

Jeff H. Mettler

University of Kentucky, jeffrey.h.mettler@gmail.com

Digital Object Identifier: http://dx.doi.org/10.13023/ETD.2016.368

Right click to open a feedback form in a new tab to let us know how this document benefits you.

\section{Recommended Citation}

Mettler, Jeff H., "THE EFFECTS OF A HIP FLEXOR STRETCHING PROGRAM ON RUNNING KINEMATICS IN INDIVIDUALS WITH LIMITED PASSIVE HIP EXTENSION" (2016). Theses and Dissertations--Kinesiology and Health Promotion. 35.

https://uknowledge.uky.edu/khp_etds/35

This Master's Thesis is brought to you for free and open access by the Kinesiology and Health Promotion at UKnowledge. It has been accepted for inclusion in Theses and Dissertations--Kinesiology and Health Promotion by an authorized administrator of UKnowledge. For more information, please contact UKnowledge@lsv.uky.edu. 


\section{STUDENT AGREEMENT:}

I represent that my thesis or dissertation and abstract are my original work. Proper attribution has been given to all outside sources. I understand that I am solely responsible for obtaining any needed copyright permissions. I have obtained needed written permission statement(s) from the owner(s) of each third-party copyrighted matter to be included in my work, allowing electronic distribution (if such use is not permitted by the fair use doctrine) which will be submitted to UKnowledge as Additional File.

I hereby grant to The University of Kentucky and its agents the irrevocable, non-exclusive, and royalty-free license to archive and make accessible my work in whole or in part in all forms of media, now or hereafter known. I agree that the document mentioned above may be made available immediately for worldwide access unless an embargo applies.

I retain all other ownership rights to the copyright of my work. I also retain the right to use in future works (such as articles or books) all or part of my work. I understand that I am free to register the copyright to my work.

\section{REVIEW, APPROVAL AND ACCEPTANCE}

The document mentioned above has been reviewed and accepted by the student's advisor, on behalf of the advisory committee, and by the Director of Graduate Studies (DGS), on behalf of the program; we verify that this is the final, approved version of the student's thesis including all changes required by the advisory committee. The undersigned agree to abide by the statements above.

Jeff H. Mettler, Student

Dr. Michael Pohl, Major Professor Dr. Heather Erwin, Director of Graduate Studies 
THE EFFECTS OF A HIP FLEXOR STRETCHING PROGRAM ON RUNNING KINEMATICS IN INDIVIDUALS WITH LIMITED PASSIVE HIP EXTENSION

THESIS

A thesis submitted in partial fulfillment of the requirements for the degree of

Master of Science in the College of Education at the University of Kentucky

By

Jeffrey Howard Mettler

Lexington, Kentucky

Director: Dr. Michael Pohl, Professor of Kinesiology

Lexington, Kentucky

2016

Copyright (C) Jeffrey Howard Mettler 2016 


\section{ABSTRACT OF THESIS}

THE EFFECTS OF A HIP FLEXOR STRETCHING PROGRAM ON RUNNING KINEMATICS IN INDIVIDUALS WITH LIMITED PASSIVE HIP EXTENSION

INTRODUCTION: Tightness of the hip flexor muscle group may contribute to altered sagittal plane kinematics of the lumbo-pelvic-hip (LPH) complex during dynamic movements. Therefore, the purpose of this study is to analyze the effects of a three-week home-based stretching program on passive hip extension (PHE), as well as on active hip extension (AHE), anterior pelvic tilt (APT), and lumbar spine extension (LSE) when running. METHODS: Twenty healthy subjects with limited PHE underwent a 3D gait analysis both prior (PRE) and following (POST) a three-week static hip flexor stretching program. RESULTS: Following the stretching program, peak PHE increased significantly ( $\mathrm{P}<0.001)$, while no significant improvements were reported in AHE, APT, or LSE. In addition, no relationship was found between the change in PHE with either the change in AHE, APT, or LSE. Finally, a high relationship was observed between AHE and APT during running $(r=0.83, p<0.001)$, and low relationships were observed between APT and LSE $(r=-0.41, p=0.08)$ and AHE and LSE $(r=-0.34, p=0.15)$. CONCLUSION: A three-week static stretching program of the hip flexor musculature resulted in an increase in PHE, but the sagittal plane kinematics of the LPH complex during running remained unchanged. The correlations observed between AHE, APT, and LSE suggest there is a kinematic relationship between the hip, pelvis, and spine.

KEYWORDS: Running, Biomechanics, Hip, Flexibility, Kinematics

Jeffrey Mettler July 29, 2016 
THE EFFECTS OF A HIP FLEXOR STRETCHING PROGRAM ON RUNNING KINEMATICS IN INDIVIDUALS WITH LIMITED PASSIVE HIP EXTENSION

\author{
By \\ Jeffrey Howard Mettler
}

Dr. Michael Pohl Director of Thesis

Dr. Heather Erwin

Director of Graduate Studies

July 29, 2016 


\section{ACKNOWLEDGMENTS}

The following thesis, while an individual work, benefitted greatly from several key individuals. First of all, my Thesis Chair, Dr. Michael Pohl, was with me every step of the way. He provided me with guidance and feedback throughout the entire process. This project would not have been a success without his assistance. Next, I would like to thank the other members of my committee: Dr. Brian Noehren and Dr. Robert Shapiro. Both provided insight and feedback that greatly improved the quality of the final product. Finally, I would like to thank anyone who assisted me with data collections, specifically Ethan Stewart. This project would not have been completed without their help. 


\section{TABLE OF CONTENTS}

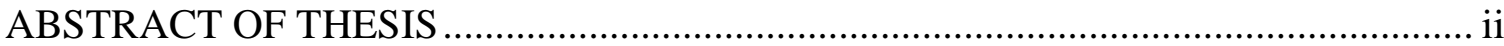

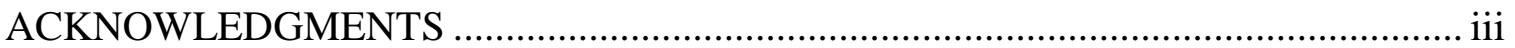

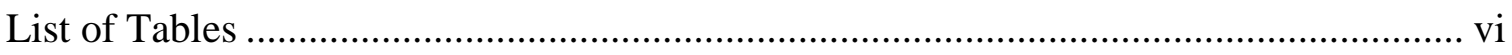

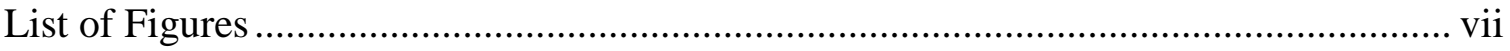

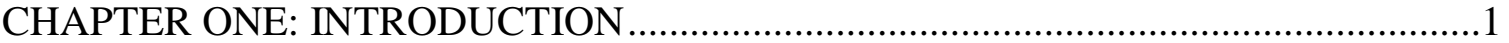

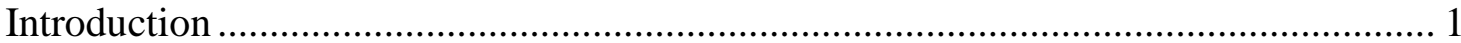

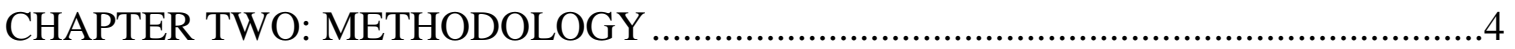

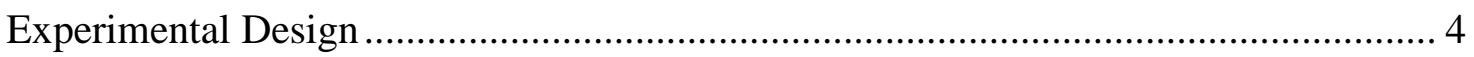

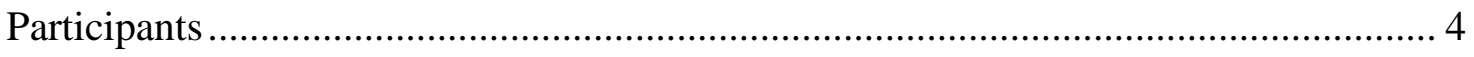

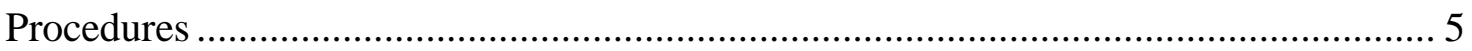

Passive Hip Extension Flexibility Measurement ..................................................... 6

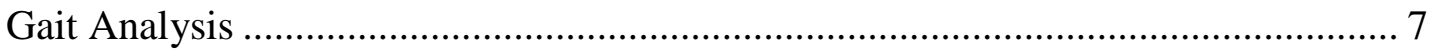

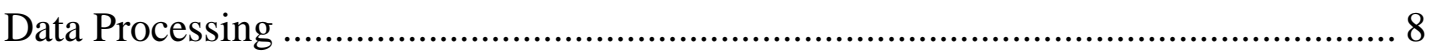

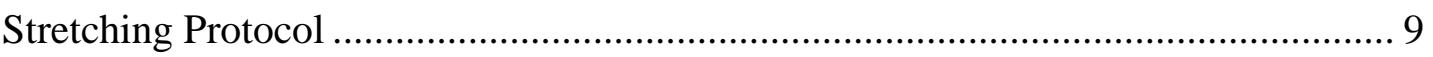

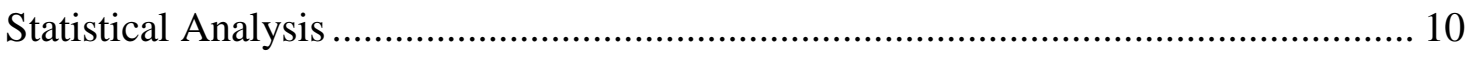

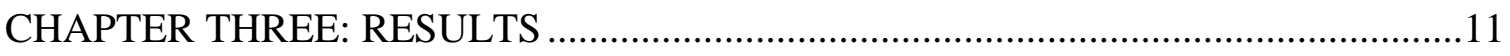

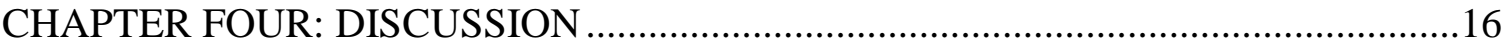

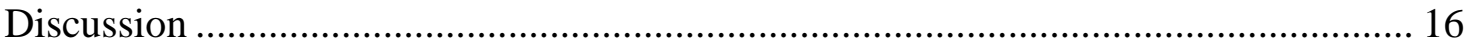

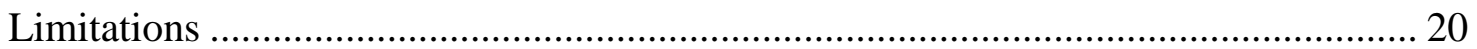

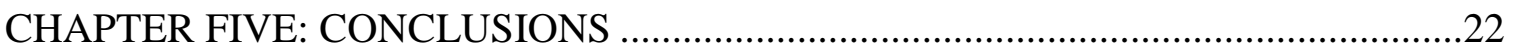

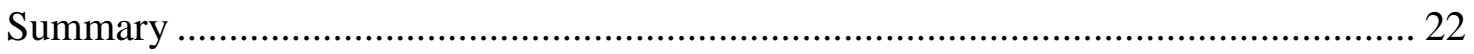

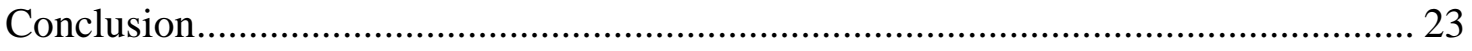

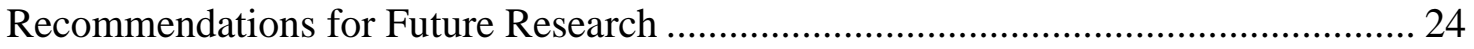

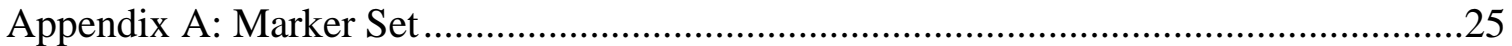

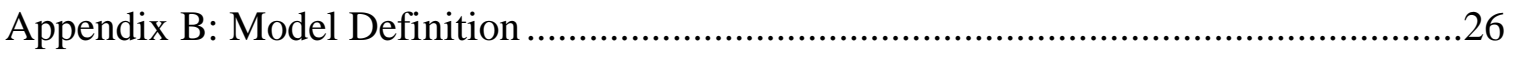

Appendix C: Expanded Literature Review ...........................................................28

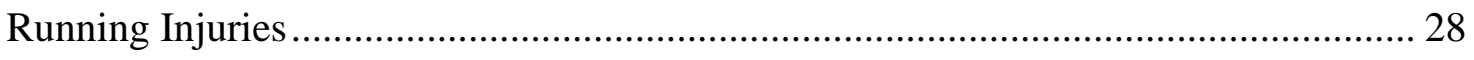

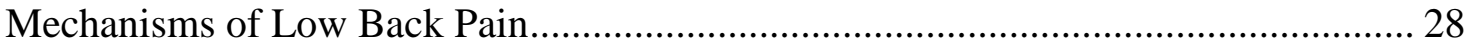

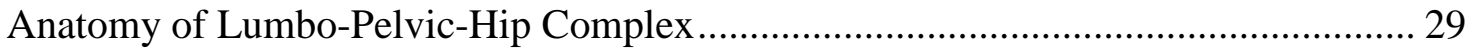

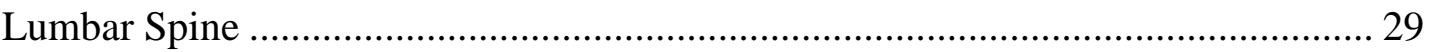

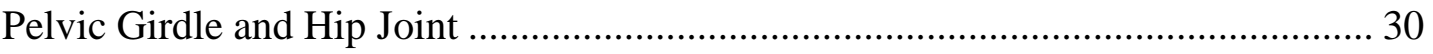




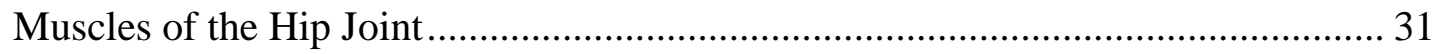

Kinematics of Lumbo-Pelvic-Hip Complex and Low Back Pain ................................. 32

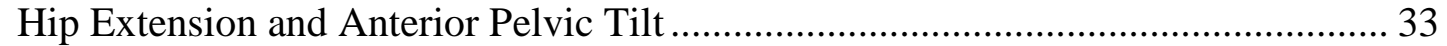

Anterior Pelvic Tilt and Lumbar Spine Extension ................................................ 33

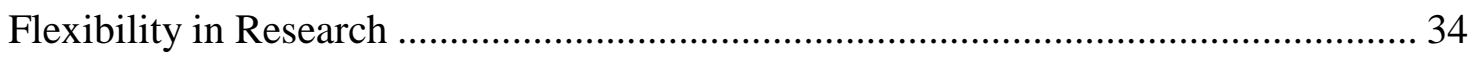

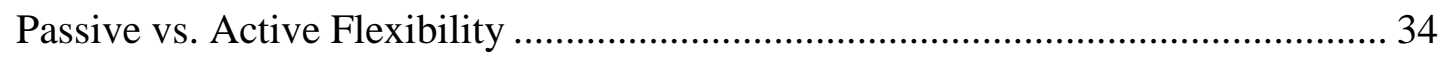

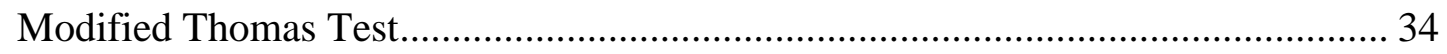

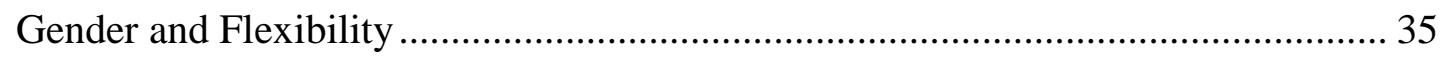

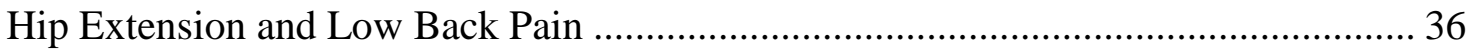

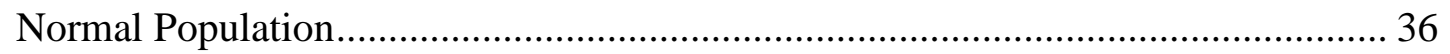

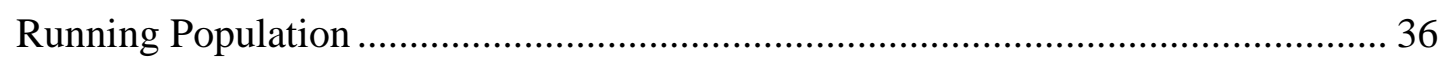

Improving Flexibility to Reduce the Symptoms of Low Back Pain ........................... 37

Frequency and Duration of Static Stretching .................................................... 37

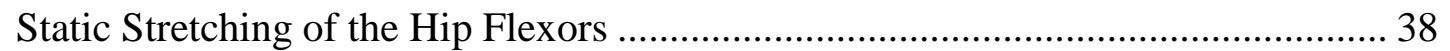

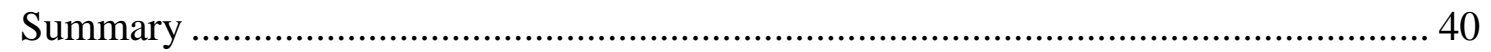

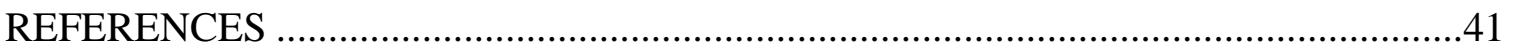

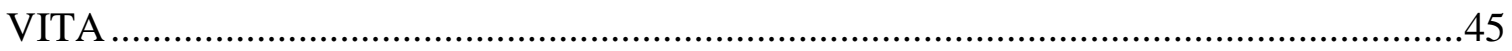




\section{List of Tables}

Table 3.1: Passive Flexibility and Kinematic Values (Mean \pm SD) both prior to (PRE) and following (POST) the three-week stretching program .....................11

Table 3.2: Relationships between change in passive hip extension (PRE-POST) and the changes in several kinematic variables during running (PRE-POST).........13 


\section{List of Figures}

Figure 2.1: Modified Thomas Test ............................................

Figure 2.2: Stretching Program .......................................................

Figure 3.1: Individual changes in passive hip extension flexibility following the three

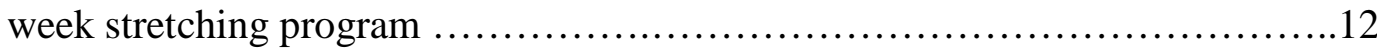

Figure 3.2: Mean ensemble curves of active hip extension, anterior pelvic tilt, and

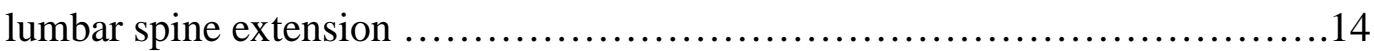

Figure 3.3: Relationships between sagittal plane kinematics of the LPH complex .....15 


\section{CHAPTER ONE: INTRODUCTION}

The introduction section provides background information about the topics of low back pain and hip extension flexibility in runners. It justifies the importance of conducting this study.

\section{Introduction}

The popularity of running is undeniable, with over 50 million people participating each year in the United States alone (1). Unfortunately, participation in running is often accompanied by injury. Running injuries are common, with annual incidence rates ranging from between $19.4 \%$ to $92.4 \%$, depending on the specific definition of a "running injury" (2). Generally, it is accepted that approximately 50\% of runners experience an injury each

year (3). According to Lopes et al. (4), low back pain accounts for approximately 5.5\% of those injuries.

Although the exact underlying cause of low back pain in runners is currently unknown, research has demonstrated that individuals with low back pain exhibit a reduction in passive hip extension range of motion compared to asymptomatic individuals $(5,6)$, which may translate into reduced active hip extension during dynamic movements. Reduced active hip extension flexibility has been correlated to greater anterior pelvic tilt during running $(7,8)$. In turn, greater anterior pelvic tilt has been associated with greater extension of the lumbar spine during running (9). Since it is postulated that excessive lumbar spine extension may contribute to low back pain, the kinematic relationship between hip, pelvis, and lumbar spine, also known as the lumbo-pelvic-hip (LPH) complex, provides a potential mechanism to help explain low back pain during running. 
Limited passive hip extension flexibility has been associated with low back pain in runners, so it is possible that reduced flexibility of the hip flexor muscle group may contribute to the reduction in hip extension during running. In an attempt to explain low back pain in runners, Bach et al. (10) compared hip flexibility between runners and nonrunners. They found reduced passive flexibility of the hip flexors and hip extensors in the runners. Furthermore, the researchers separated the runners into a back pain group and a healthy group in order to further explore the relationship between low back pain and hip flexor tightness. While they were not able to correlate the reduced flexibility with low back pain, their small sample size $(\mathrm{N}=10)$ limited the conclusions of their study, and thus further investigation into the relationship between hip flexor flexibility and low back pain is warranted.

Stretching the hip flexors to improve the range of motion at the hip may be a useful intervention in terms of reducing low back pain. Winters et al. (11) found that six weeks of either passive or active stretching of the hip flexors was effective at increasing passive hip extension flexibility. Moreside \& McGill (12) reported similar results when they also implemented a six week passive stretching program in individuals with limited passive hip extension flexibility. However, in a follow-up study, the same aforementioned authors (13) found that improvements in passive flexibility at the hip flexors did not necessarily result in improvements in active hip extension flexibility during several functional dynamic movement tasks. However, no study has yet examined the effects of static hip flexor stretching on active hip extension during running.

Research suggests that reduced passive hip extension flexibility may put an individual at risk for low back pain by decreasing active hip extension flexibility while 
increasing both anterior pelvic tilt and lumbar spine extension during running. Therefore, the purpose of this study is to analyze the effects of a three week stretching program on passive hip extension flexibility, as well as on the sagittal plane kinematics of the hip, pelvis, and spine during running. It is hypothesized that greater passive and active hip extension flexibility will be measured following the three week stretching program. It is also hypothesized that greater passive and active hip extension flexibility will be accompanied by reductions in both anterior pelvic tilt and lumbar spine extension. 


\section{CHAPTER TWO: METHODOLOGY}

The methodology section details the specific steps that were taken to conduct this study. It contains information regarding the research design, population of interest, instrumentation, data collection procedures, and how the data were analyzed.

\section{Experimental Design}

This was a laboratory based, one-group, pretest-posttest, pre-experimental design. The independent variable was a three-week static stretching program. The dependent variables included passive peak hip extension during the modified Thomas test, as well as peak hip extension, anterior tilt, and lumbar spine extension during running.

\section{Participants}

Participants were recruited using convenience sampling from the university campus. Physical and online flyers as well as word of mouth were utilized to recruit. Participants were between the ages of 18-40 and regularly engaged in physical activity involving running for at least 30 minutes, three times per week. Subjects were excluded if they were; pregnant; currently experiencing any pain in the lower extremities or spine; had suffered an injury to the lower extremity or spine that limited activity in the past three months; had undergone major surgery to the lower extremity or spine at any time; or if they were not comfortable running on a treadmill without the use of handrails. Finally, in order to participate, subjects were required to exhibit limited passive hip extension flexibility based on screening measurements.

Based on a reported $4.1^{\circ}\left(\mathrm{SD}=7.5^{\circ}\right)$ increase in active hip extension flexibility when walking following a stretching program (14), we estimated a $5^{\circ}$ increase when 
running (due to greater joint range of motion during running). An a priori sample size of 19 was calculated using an alpha level of 0.05 , a power of 0.8 , and an estimate of the change in hip extension of $5^{\circ}$. Participants were recruited until the required sample size had completed the protocol. A total of 36 participants were screened for the study. Of those screened, 24 met the inclusion criteria of limited passive hip extension flexibility. A total of 4 subjects were excluded from the study since they failed to complete the flexibility protocol and/or follow-up testing session: two subjects because of busy schedules, one subject due to a sprained ankle, and one subject failed to follow the stretching program. Therefore, 20 participants $(27.80 \pm 5.33$ years, $1.71 \pm 0.06 \mathrm{~m}, 67.62 \pm 11.44 \mathrm{~kg})$ consisting of 11 females (27.82 \pm 5.95 years, $1.69 \pm 0.06 \mathrm{~m}, 62.17 \pm 8.69 \mathrm{~kg})$ and 9 males $(27.78 \pm$ 4.82 years, $1.74 \pm 0.06 \mathrm{~m}, 74.27 \pm 11.22 \mathrm{~kg}$ ) adhered to the stretching program and completed the entire study.

\section{Procedures}

Participants were required to complete one screening visit to establish whether they met the inclusion criteria for limited passive hip extension. Prior to the screening session, all procedures were explained to the potential participants and they were asked to provide informed consent using a form approved by the Institutional Review Board. During the screening session, participants underwent a passive hip extension flexibility assessment using the modified Thomas Test (MTT) to determine whether they met the inclusion criteria. Participants were considered to have limited hip extension flexibility if they produced a flexibility value of $-1.5^{\circ}$ or greater. This value was based on hip flexibility measurements of a previous study where the $50^{\text {th }}$ percentile of a sample of 77 healthy individuals aged 18-35 had a passive hip extension measurement of $-1.5^{\circ}\left(\mathrm{SD}=6^{\circ}\right)(15)$. 
Participants who qualified for the study based on the inclusionary/exclusionary criteria as well as the results of the preliminary screening completed two data collection sessions, separated by three weeks of static stretching. All data were collected by a single investigator to avoid inter-tester variability. Basic demographic data, including height, weight, age, sex, and current physical activity level were obtained during the baseline visit. Passive hip extension measurements were recorded and a gait analysis was conducted during both the baseline (PRE) and follow-up (POST) sessions. A stretching protocol education session was held following the baseline session to teach participants the homebased stretching program that they were to perform for three weeks. Both data collections occurred in the Biomotion Laboratory (MDS Room B04A) at the University of Kentucky and lasted approximately 90 minutes each. Participants were asked to wear sport clothing (shorts and a tank top/sports bra).

\section{Passive Hip Extension Flexibility Measurement}

The modified Thomas test (MTT) was used to assess passive hip extension range of motion on both limbs. The MTT has been found to possess good reliability (16). To test the intra-rater reliability of the primary researcher of this study, a reliability analysis was

performed between the measurements of the MTT performed during both the screening session and the PRE session. The analysis produced a Standard Error of Measurement (SEM) of $3.5^{\circ}$.

To perform the MTT, participants were positioned on their back with their measured hip at the edge of the treatment table and their tested leg hanging passively off the table (Figure 2.1). At the same time, the opposite leg was held by the participant with the hip and knee in a flexed position against the chest. Participants were instructed to pull 
their knee straight to their head in order to prevent any hip abduction. Care was also taken to ensure that the participant's low back laid flat against the table so that the pelvis and lumbar spine remained in a neutral position throughout the entire test. Using a digital inclinometer placed on the thigh, the passive hip extension range of motion was measured. Measurements were considered negative if the thigh dropped below the horizontal and positive if the thigh remained above the horizontal.

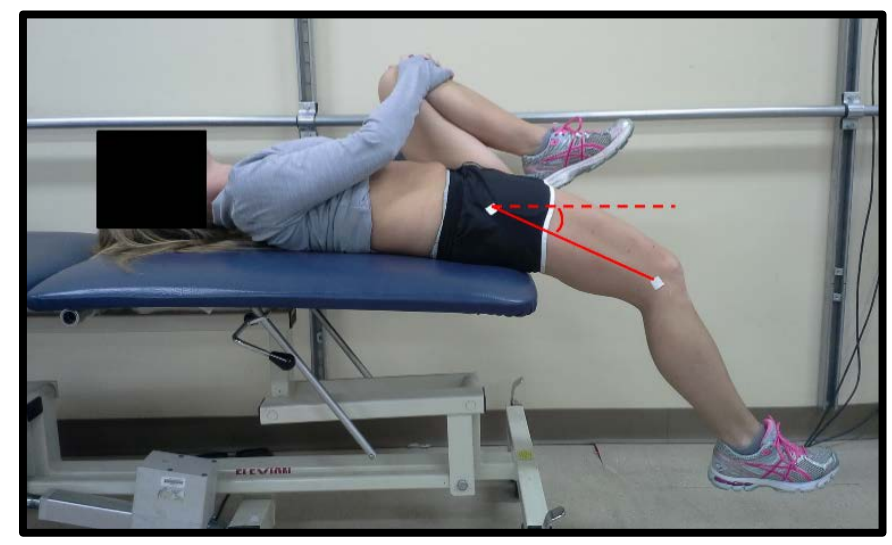

Figure 2.1: The modified Thomas test (MTT) has the participant lie on the edge of a table with the tested leg hanging passively off the table and the opposite leg held against the chest.

\section{Gait Analysis}

A standardized neutral running shoe (New Balance, R662WSB, Boston, MA) was provided for each participant to wear during the data collection. Retro-reflective markers were placed bilaterally on the following landmarks: acromion process, iliac crest, ASIS, PSIS, greater trochanter, medial and lateral knee, medial and lateral malleoli, lateral heel, proximal heel, distal heel, first and fifth metatarsal head, and toe. Additional markers were placed on the sternal notch, seventh spinous vertebral process (C7), apex of kyphosis (T6 or T7, depending on the person), apex of lordosis (L3), and an offset on the right foot. 
Finally, rigid body clusters of 4-5 markers were placed on the posterior aspect of the subject's right and left thigh and shank (Appendix A).

The gait analysis was completed using 10 Eagle Motion Analysis cameras (Motion Analysis Corp, Santa Rosa, CA) and an instrumented treadmill (Bertec, Columbus, OH) recording at $200 \mathrm{~Hz}$ and $1000 \mathrm{~Hz}$ respectively. A static trial of each participant was captured to identify the anatomical locations of the markers. Following the static trial, some of the anatomical markers were removed, leaving only the 36 tracking markers. Participants were told to select a speed on the treadmill equivalent to an easy 30 minute run. After a five minute acclimation period (17), marker trajectory data were collected for three trials of 10 seconds in duration. The average running speed for the 20 participants was $2.85 \pm 0.38 \mathrm{~m} / \mathrm{s}$.

\section{Data Processing}

Marker trajectory data were tracked using Cortex software (Motion Analysis Corp., Santa Rosa, CA). Further data processing, including filtering and calculating joint/segment angles, was conducted using Visual 3D software (C-Motion Inc., Germantown, MD). Raw marker trajectory data were filtered using a fourth order low-pass Butterworth filter with a cut-off frequency of $8 \mathrm{~Hz}$. An X-Y-Z cardan sequence (sagittal-frontal-transverse) was used to quantify joint angles, in which the distal segment was expressed relative to the proximal segment. The pelvis was expressed relative to the lab. For the kinematic description of lumbar spine extension, an adapted version of the marker set and model from Junquiera et al. (18) was utilized. The only difference in relation to the marker set was that a virtual marker at the midpoint of the two PSIS markers was used instead of a marker at the lower edge of the sacrum (S2 or S3). Kinematic data of the least flexible limb based on the results of the MTT was selected for further analysis. Initial contact and toe-off were 
identified using vertical ground reaction force data, with the threshold set at 35 N. Discrete variables of interest included peak values for hip extension, anterior pelvic tilt, and lumbar spine extension. The peak values for the kinematic variables of interest were defined as the greatest values observed during the gait cycle. The ensemble mean value for five consecutive gait cycles of each kinematic variable was calculated for each subject.

\section{Stretching Protocol}

All participants were assigned the same stretching protocol, which consisted of a standing hip flexor stretch and a kneeling modified lunge stretch (Figure 2.1). Ten repetitions were completed for each stretch, with a 10 second hold and a 10 second rest for each repetition. Participants were asked to perform this stretching protocol once a day for the duration of the three-week study. Written instructions were provided to each participant, describing the stretching techniques and the program details. After explaining the procedures, the stretch was demonstrated by the researcher. The participant was then asked to perform the stretch in the presence of the researcher to ensure proper technique. They were encouraged to maintain their daily activities, with the addition of the stretching protocol as the only exception. Also, a daily calendar was given to each participant, which allowed them to check off each day after they completed their daily stretches. The primary investigator checked in with participants at the end of each week in order to monitor and encourage compliance. Adherence was quantified as a percentage, and was calculated as the number of days stretched divided by the total number of days multiplied by 100 . While they were encouraged to stretch every day, participants were required to stretch at least five days each week (71.4\%) for adherence to the program to be met. For the 20 participants, adherence was found to be $86.7 \pm 9.0 \%$. 


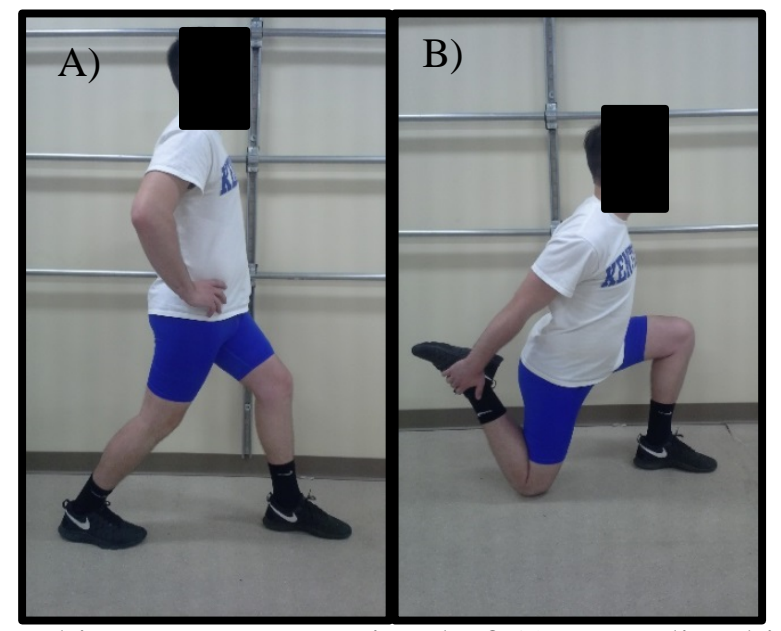

Figure 2.2: The stretching program consisted of A) a standing hip flexor stretch and B) a modified kneeling lunge stretch, which were performed each day.

\section{Statistical Analysis}

All kinematic and flexibility dependent variables of interest were compared before and after the three-week stretching program using dependent t-tests. Correlations were performed between the change in passive hip extension and: i) the change in active hip extension; ii) the change in anterior pelvic tilt; iii) the change in lumbar spine extension. In addition, correlations were conducted between selected peak sagittal plane kinematic values of the LPH complex during running (using the PRE session), including active hip extension vs. )anterior pelvic tilt, active hip extension vs. lumbar spine extension, and anterior pelvic tilt vs. lumbar spine extension. Finally, correlation analyses were conducted for stretching adherence vs. the change in passive hip extension as well as stretching adherence vs. the change in active hip extension. Interpretation of Pearson correlation coefficient (r) followed guidelines set out by Hinkle et al. (19) for very high ( $r>0.90)$, high $(r=0.70-0.90)$, moderate $(r=0.50-0.69)$, low $(r=0.30-0.49)$, and negligible $(r$ $=0.00-0.29)$. All statistical analyses were performed using SPSS statistical software (SPSS Inc., Chicago, IL) with a significance level of $\mathrm{p}<0.05$. 


\section{CHAPTER THREE: RESULTS}

The results section presents the findings of this study, including the dependent ttests and the correlation analyses that were conducted on the data collected.

\section{Results}

Mean values for passive hip extension, as well as sagittal plane kinematics of the LPH complex, are presented in Table 3.1. The results of the study showed a significant increase in passive hip extension following the three-week static stretching program (Table 3.1). In addition, Figure 3.1 shows the individual changes in passive hip extension that each participant made following the three-week stretching program, with 19 out of 20 participants demonstrating an increase in passive hip extension (range $=-8.3^{\circ}$ to $24.0^{\circ}$ ).

Table 3.1: Passive Flexibility and Kinematic Values (Mean \pm SD) both prior to (PRE) and following (POST) the three-week stretching program

\begin{tabular}{lllll}
\hline & PRE & POST & P-value & $\begin{array}{l}\text { Effect } \\
\text { Size }\end{array}$ \\
& & & & \\
\hline $\begin{array}{l}\text { Passive Flexibility ( }{ }^{\circ} \text { ) } \\
\text { Hip Extension (-ve) }\end{array}$ & $9.4 \pm 5.2$ & $-1.2 \pm 8.9$ & $<0.001^{*}$ & 0.59 \\
Gait Kinematics ( ${ }^{\circ}$ ) & & & & \\
$\quad$ Hip Extension (-ve) & $-4.3 \pm 6.2$ & $-4.1 \pm 5.9$ & 0.75 & 0.02 \\
$\quad \begin{array}{l}\text { Anterior Pelvic Tilt (+ve) } \\
\text { Lumbar Spine Extension }(-\end{array}$ & $-21.0 \pm 4.7$ & $21.6 \pm 4.6$ & 0.36 & 0.06 \\
ve) & & $-25.5 \pm 8.4$ & 0.68 & 0.02 \\
\hline
\end{tabular}

* Denotes significance at $\mathrm{P}<0.05$ level

Despite the significant improvement in passive hip extension following the threeweek stretching program, there were no differences in peak active hip extension, anterior 
pelvic tilt, or lumbar spine extension during running between PRE and POST (Table 3.1). The sagittal plane kinematics of hip extension (Figure 3.2a), anterior pelvic tilt (Figure 3.2b), and lumbar spine extension (Figure 3.2c) remained nearly identical through the entire gait cycle following the three-week stretching program. In addition, no relationship was found between the change (between PRE and POST) in passive hip extension with either the change in peak active hip extension, peak anterior pelvic tilt, or peak lumbar spine extension during running (Table 3.2).

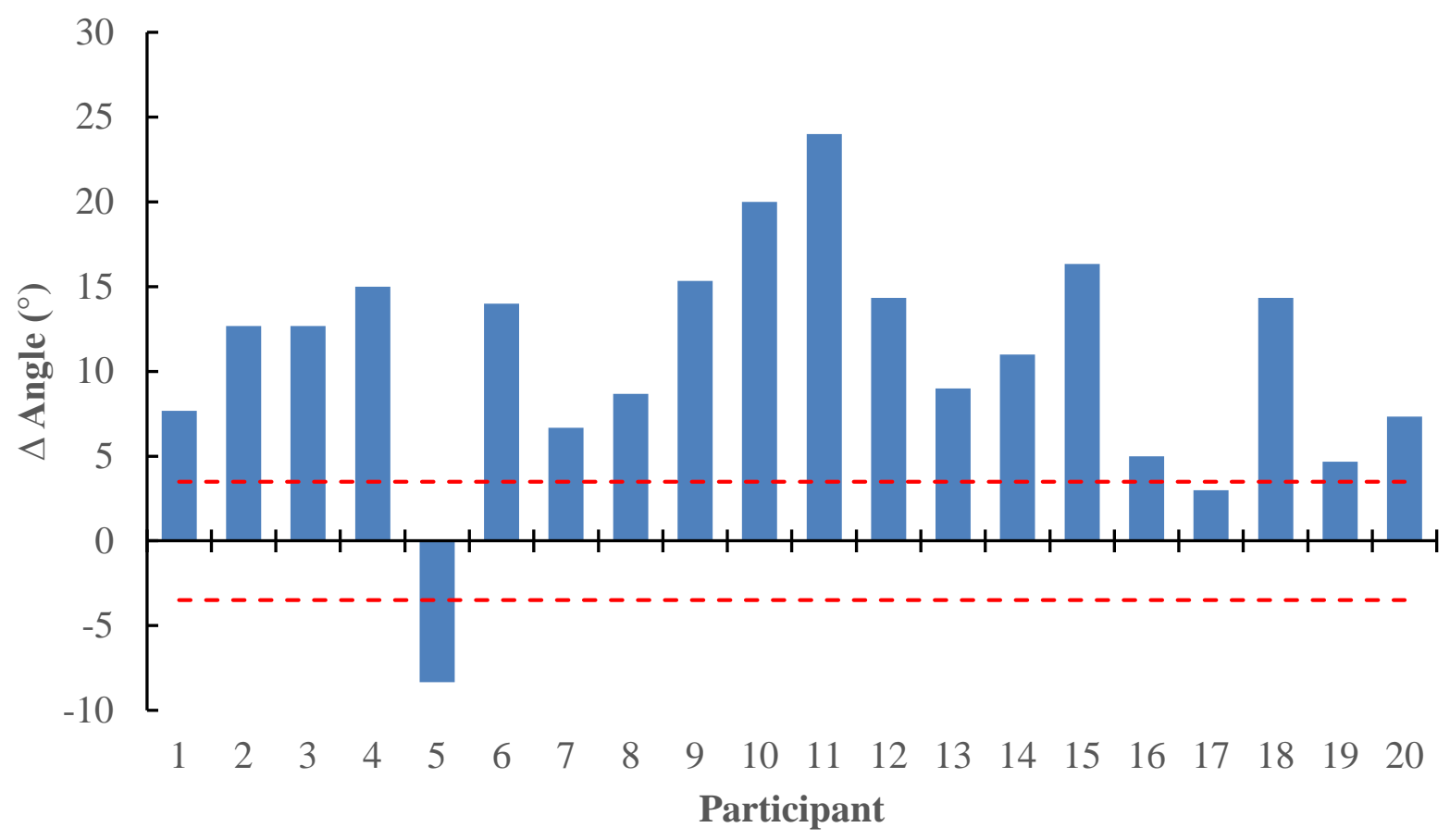

Figure 3.1: Individual changes in passive hip extension flexibility following the three week stretching program. Positive bars indicate improvements in flexibility, while negative bars indicate reductions. The dashed lines indicate the precision of the flexibility measurement as determined by the intra-rater reliability analysis. 
Table 3.2: Relationships between the change in passive hip extension (PRE-POST) and the changes in several kinematic variables during running (PRE-POST)

\begin{tabular}{lll}
\hline & Pearson $\mathbf{r}$ & P-value \\
\hline Active Hip Extension & -0.26 & 0.27 \\
Anterior Pelvic Tilt & 0.21 & 0.38 \\
Lumbar Spine Extension & -0.40 & 0.08 \\
\hline
\end{tabular}

While no relationship was found between passive hip extension and the sagittal plane kinematics of the LPH complex, there was a high positive correlation $(r=0.83, p<$ 0.001) between anterior pelvic tilt and active hip extension during running (Figure 3.3a), where a greater peak hip extension angle was associated with a reduced anterior pelvic tilt angle. A low negative correlation was found between lumbar spine extension and anterior pelvic tilt $(r=-0.41, p=0.08)$, showing that as the peak anterior pelvic tilt angle increased, the peak lumbar spine extension angle increased as well (Figure 3.3b). Lumbar spine extension and hip extension also displayed a low negative correlation $(r=-0.34, p=0.15)$, where a greater peak hip extension angle was associated with a lower peak lumbar spine extension angle (Figure 3.3c).

Finally, there were no relationships between stretching adherence and change in passive hip extension ( $r=-0.176, p=0.457)$ or between stretching adherence and change in peak active hip extension angle $(r=0.019, \mathrm{p}=0.937)$. 
A)

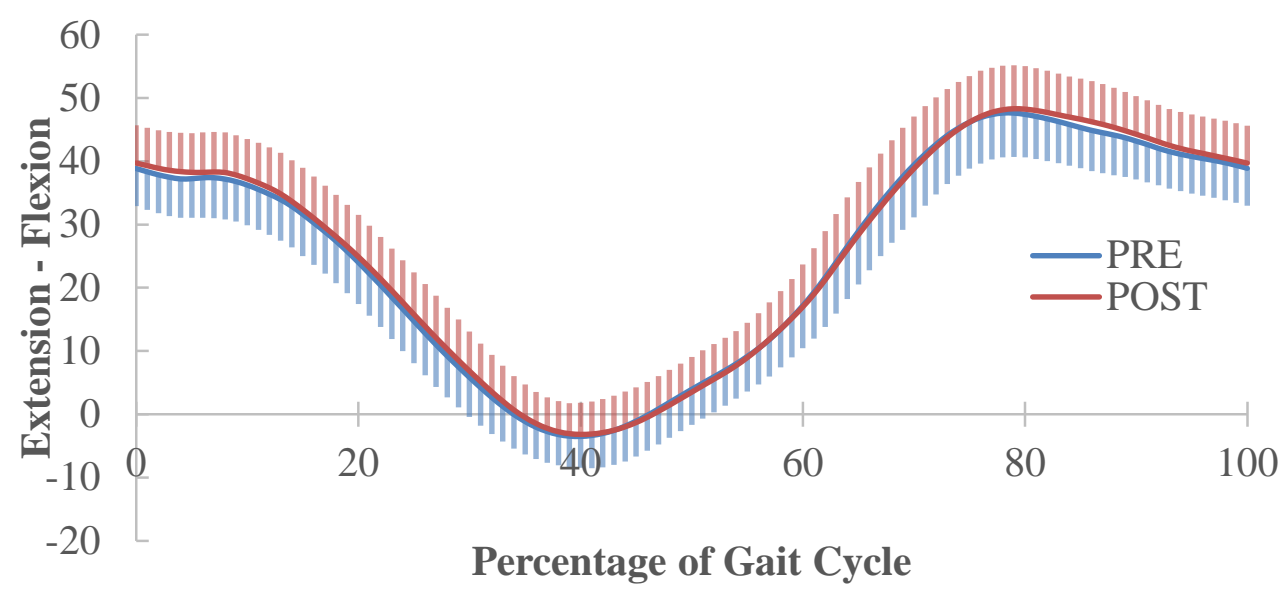

B)

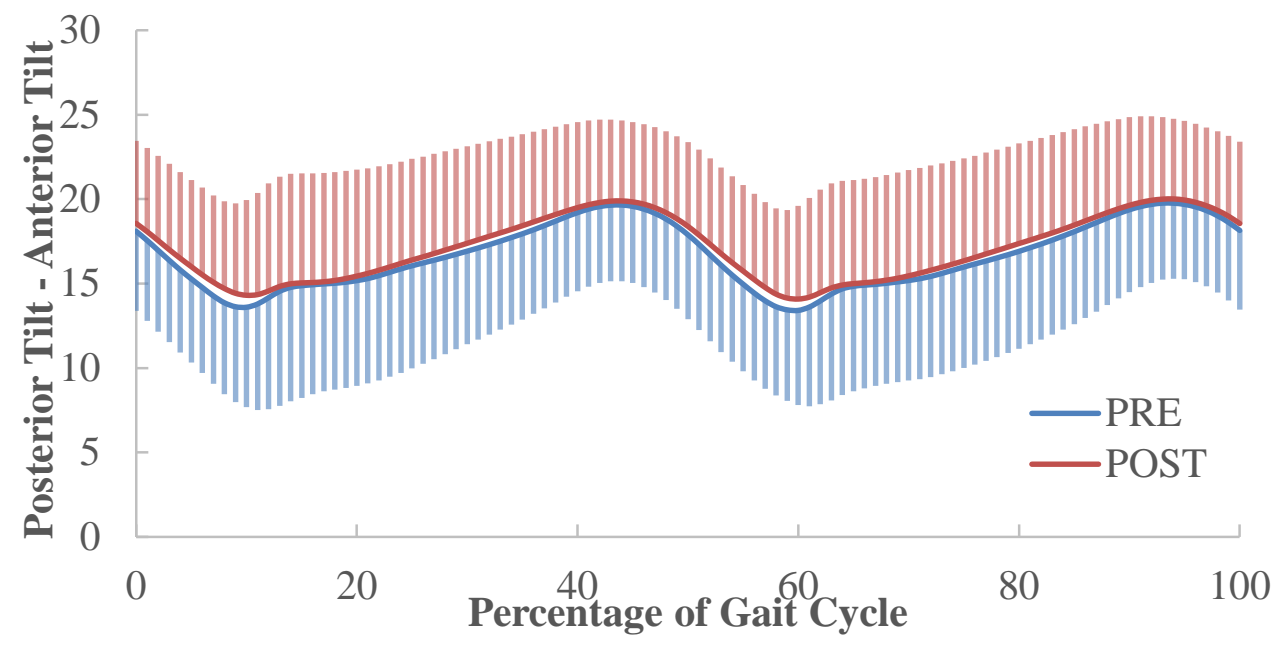

C)

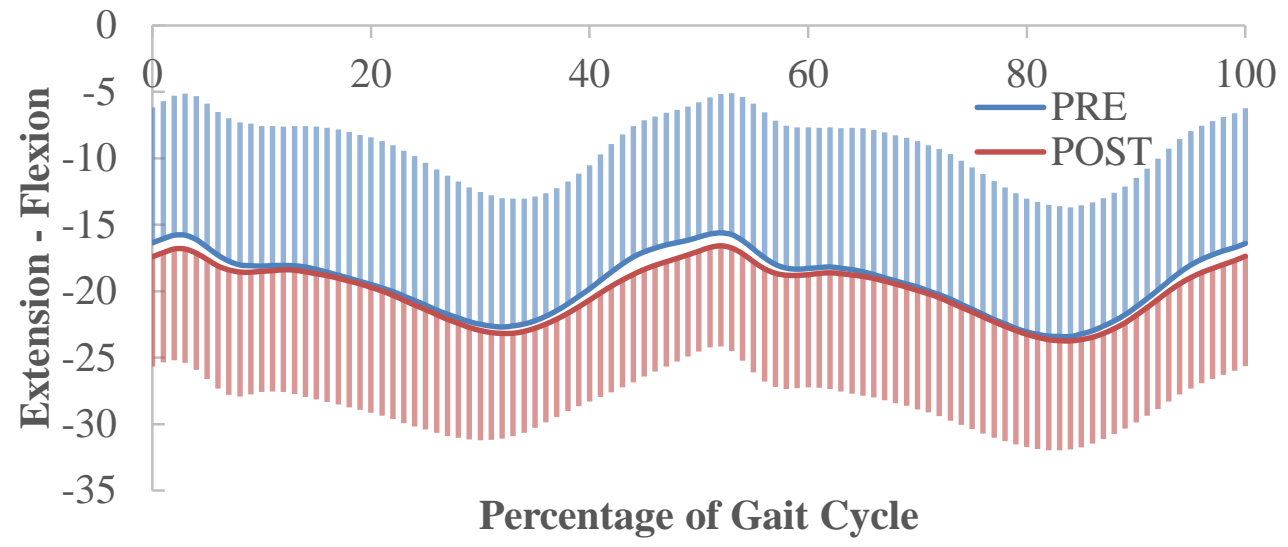

Figure 3.2: Mean ensemble curves of A) active hip extension; B) anterior pelvic tilt; and C) lumbar spine extension, throughout the entire gait cycle. 
A)

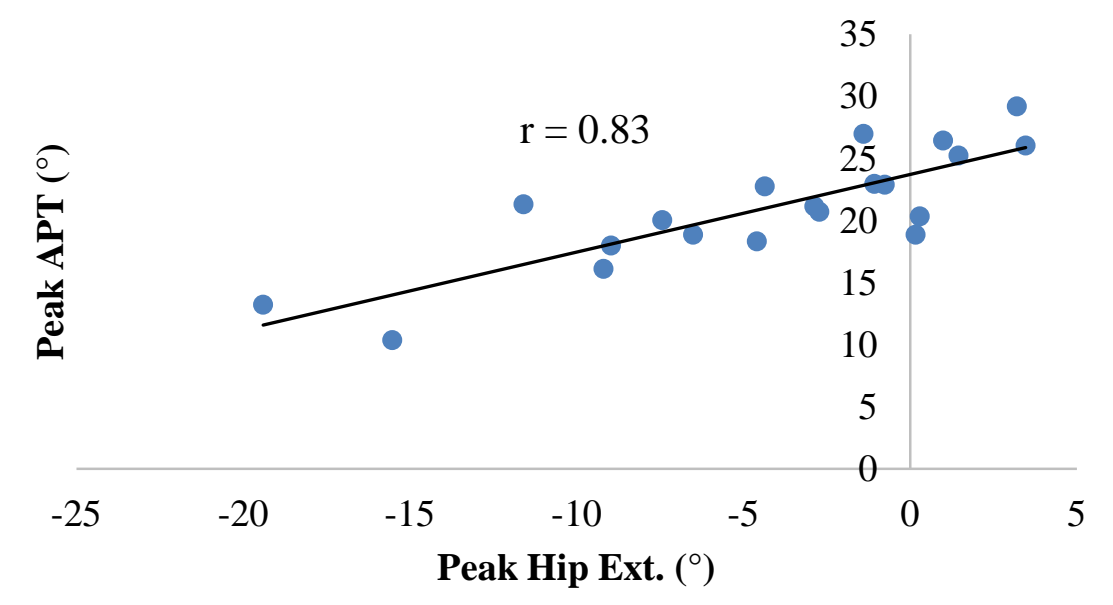

B)
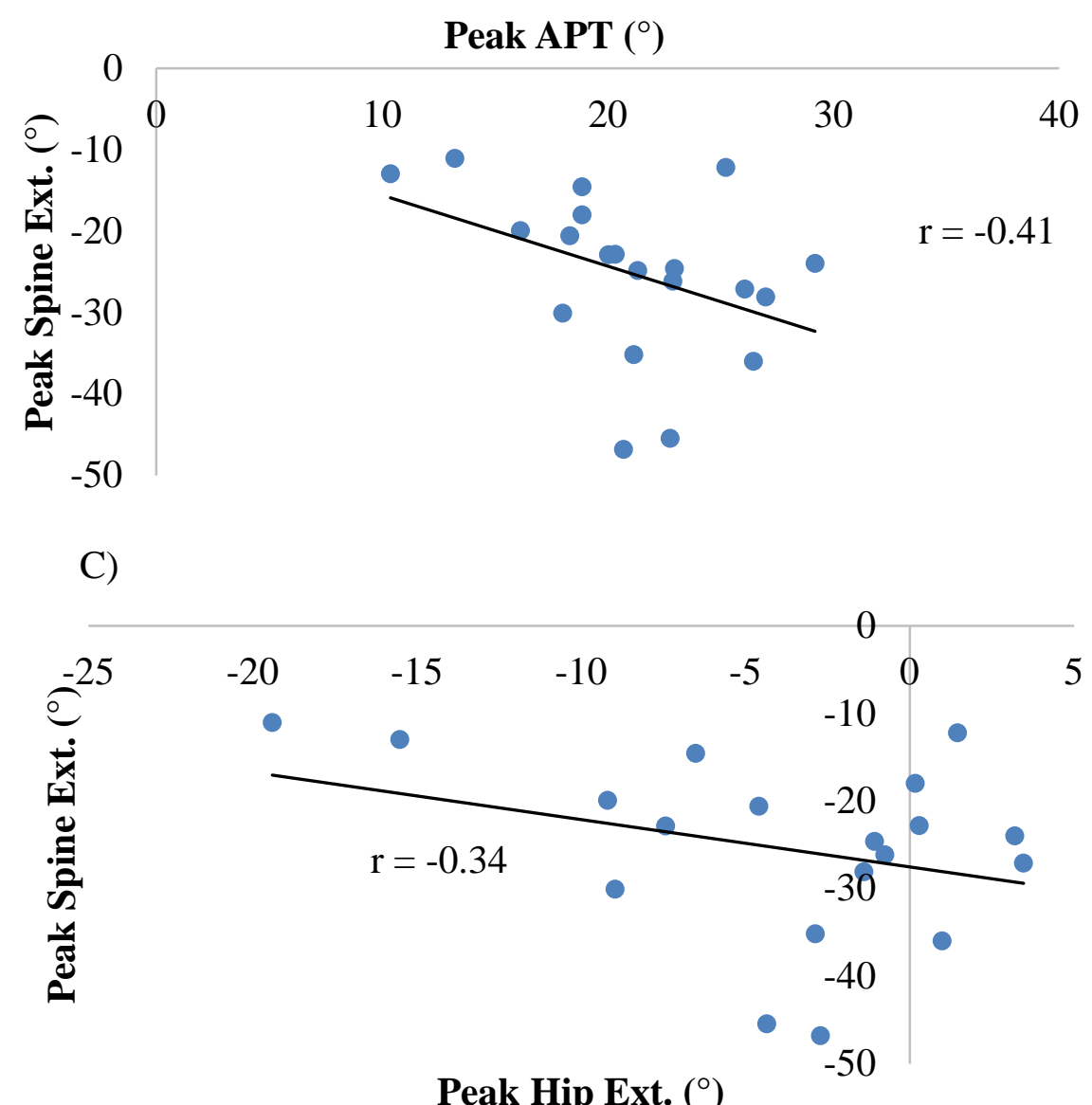

Figure 3.3: Relationship between: A) peak anterior pelvic tilt (APT) and peak hip extension during running; B) peak lumbar spine extension and peak anterior pelvic tilt (APT) during running; C) peak lumbar spine extension and peak hip extension during running. 


\section{CHAPTER FOUR: DISCUSSION}

The discussion section interprets the results that were reported in chapter three. It also includes a listing of the potential limitations of the study. The discussion aims to contribute new knowledge to the topic in question.

\section{Discussion}

The aim of this study was to investigate the effects of a three week stretching program on passive hip extension flexibility and the sagittal plane kinematics of the LPH complex during running. An increase in passive hip extension was observed following the stretching program. However, contrary to our hypothesis we observed no increase in active hip extension or reductions in anterior pelvic tilt or lumbar spine extension during running following three weeks of static stretching.

The results we found for passive hip extension were similar to previous studies (11, 12). Winters et al. (11) reported improvements in passive hip extension of $13^{\circ}$ and $14^{\circ}$ following three weeks and six weeks of static stretching respectively. Similarly, a $13.6^{\circ}$ increase in passive flexibility was reported by Moreside and McGill (12), who analyzed passive hip extension following six weeks of static stretching in healthy young men with limited hip mobility. The improvements reported in the literature are of a similar magnitude to the change of nearly $11^{\circ}$ noted in the present study. The increase in passive flexibility can be attributed to the viscoelastic properties of muscle-tendon units. Repeated tensile loads on a muscle-tendon unit result in prolonged elongation of the muscle-tendon unit, which suggests that static stretching can improve the range of motion at a joint (20). The significant increase in passive hip extension range of motion found in the present study 
shows that the experimental protocol consisting of three weeks of static stretching was indeed successful. The fact that passive hip extension improved in 19 out the 20 participants also highlights the effectiveness of the stretching program.

Despite the increase in passive hip extension, we observed no increase in active hip extension during running following three weeks of static stretching. Furthermore, there were no reductions in anterior pelvic tilt or and lumbar spine extension during running. These results are consistent with the findings of Moreside and McGill (13), who reported no significant changes in active hip extension during several functional movements despite observing an increase in passive hip extension following six weeks of static stretching in a healthy young population. Watt et al. (14) also found that an increase in passive hip extension did not correspond to an increase in active hip extension during walking in a healthy elderly population. Thus, it appears that improvements in passive hip extension are not accompanied by changes in active hip extension in a healthy population, regardless of the activity performed (functional movement, walking, running) or the age of the sample.

Although we did not see an increase in the mean active hip extension for the sample, there appeared to be individual variation in the response to the static stretching program. For example, one participant displayed an increase in passive hip extension of $24.0^{\circ}$, while another actually decreased by $8.3^{\circ}$. Similar variation was also observed in active hip extension (range $=-10.1^{\circ}-5.5^{\circ}$ ). Therefore, a correlation analysis was conducted to determine whether individuals with greater improvements in passive hip extension also underwent greater improvements in active hip extension during running. However, no relationship was found between the changes in passive and active hip extension, thus 
providing further evidence that improvements in passive flexibility do not necessarily translate to a greater range of motion during dynamic movements.

While the extensibility of the musculature surrounding the hip joint has the potential to influence the movement pattern of the joint, the poor association found between passive and active hip extension suggests that flexibility is not the only factor influencing the dynamic range of motion at the hip. Some alternative factors that may help explain the lack of relationship between passive and active hip extension include the geometry of the joint (21) and the speed that the dynamic task is performed (7). Wilson et al. (21) showed that joints have a preferred path of least resistance based on both the geometric parameters of the articular surfaces and the surrounding tissues. The tissues and ligaments surrounding a joint do play a role in motion, but they must work together with the articular surface geometry to actually produce motion at the joint. Alternatively, Schache et al. (7) hypothesized that static flexibility may not be an important factor for a healthy population when running at submaximal speeds. Given that increases in gait speed are typically achieved by lengthening the stride, running at submaximal speeds may mean that the full range of motion at the hip is not required to perform the task. Evidence for this theory is supported by the results of Watt et al. (14), who reported that an elderly population who displayed limited active hip extension during walking underwent significant improvements in both passive and active hip extension during walking following a 10 week static stretching program. The increases in active hip extension were accompanied by an increase in stride length, which suggests that passive flexibility does play a role in dynamic movements, albeit only when those dynamic movements are pushing an individual to utilize their full range of motion. Further research is required to answer the question of 
whether passive hip extension flexibility influences active hip extension flexibility at greater running speeds.

The same self-selected speed was utilized during both the PRE and POST sessions in order to control for speed as a confounding variable. It is possible that the self-selected speed would have been different during the POST session as previous research has reported that an increase in active hip extension is accompanied by an increase in stride length (14). Keeping the running speed constant may have prevented us from seeing any changes in the sagittal plane kinematics of the LPH complex. It is possible that increasing passive hip extension flexibility would increase an individual's economy, thereby allowing them to run at a faster speed at a given effort. Therefore, it would have been helpful to collect oxygen uptake as a measure of running economy.

While no relationship was found between passive and active hip extension, several correlations were noted between the sagittal plane kinematics of the LPH complex. Our results revealed a high positive correlation between active hip extension and anterior pelvic tilt during running. A strong relationship $(r=0.90)$ between the sagittal plane kinematics of the hip and pelvis has also been reported before $(7,8)$. However, the low negative correlation we found between lumbar spine extension and anterior pelvic tilt is lower than that reported by Schache et al. (9) $(r=-0.84)$, which may be due to the different method they used to calculate lumbar extension. In addition, our results revealed a high deviation of the lumbar spine extension values throughout the entire gait cycle, thus indicating a large amount of individual variation between participants. Similar variability in lumbar spine extension was found by Whittle and Levine (22), who concluded that lumbar spine extension is a highly individualistic movement pattern. 
To our knowledge, no previous research has investigated the relationship between hip extension and lumbar spine extension during running. This is important since the relationship between the sagittal plane kinematics of the LPH complex has been discussed as a potential risk for injuries in runners (10, 23-27). Repetitive impingement of the vertebral facets due to hyperextension of the lumbar spine is thought to be related to the onset of LBP in runners (23-25). While the negative correlation found in our study was low, it still provides important information regarding the kinematic relationships between the hip, pelvis, and spine in a healthy population. Such data is vital as it provides normative data that can be used for comparison to a population with LBP.

\section{Limitations}

Several limitations should be noted in this study. Firstly, error due to marker placement is always present, although a single trained investigator performed all marker placement to avoid inter-tester variability. Further errors may be present due to skin movement artefact, as the markers placed on top of the skin do not truly represent the underlying bony landmarks. While this is an unavoidable limitation, it should be noted that physically fit nature of the participants in the study would have helped to reduce the magnitude of this error.

Adherence to the home-based stretching program is another potential limitation. While participants were given a calendar to log their progress and the primary investigator checked in at the end of each week to remind the participants of the importance of stretching, the true adherence to the stretching program was unknown. Although all but one participant appeared to follow the stretching program based on their calendar logs, it 
was assumed that participants were being honest when filling out their logs. The significant improvements in passive hip extension following the three weeks of stretching appear to support this assumption.

The reliability of the MTT has been questioned in the past (28). However, the reliability analysis performed on the MTT data from the study produced an SEM of $3.5^{\circ}$. The SEM shows that the significant difference between the PRE $\left(9.43^{\circ}\right)$ and POST $\left(-1.23^{\circ}\right)$ passive flexibility far exceeds the magnitude of the testing errors.

All participants were instructed to maintain their regular daily activities throughout the duration of the study, with the exception of adding the stretching protocol each day. However, as flexibility has been shown to increase following physical activity (29), the physical activities performed on the day of testing should have been restricted.

Furthermore, considering that all subjects were healthy, caution should be taken when relating these results to a population with LBP. It has been reported that individuals with LBP display different trunk, pelvis, and lower extremity kinematics compared to healthy individuals (30-34). While the participants of our study displayed limited passive hip extension similar to individuals who suffer from LBP, they were otherwise a healthy population. It would be of interest to conduct this study on a population of individuals with LBP to determine whether increasing passive hip extension has a beneficial effect on the sagittal plane kinematics of the LPH complex. 


\section{CHAPTER FIVE: CONCLUSIONS}

The conclusions section contains a summary of the study, as well as final conclusions that can be made based on the results of the study. Finally, recommendations are given for future studies that could be conducted.

\section{Summary}

While low back pain affects a substantial amount of runners each year, the underlying cause of it remains unknown. However, the relationship between sagittal plane kinematics of the hip, pelvis, and spine suggests that low back pain during running may be caused by limited flexibility of the hip flexor musculature. Therefore, this study analyzed the effects of a three-week hip flexor stretching program on the sagittal plane kinematics of the hip, pelvis, and spine in 20 healthy runners. Based on a screening to determine passive hip extension flexibility, individuals who exhibited limited passive hip extension were recruited to participate in two gait analyses separated by three weeks, during which they were asked to complete a home-based static stretching program of the hip flexor musculature. Following this, data from the baseline visit was compared to the data from the follow-up visit, and any potential differences in flexibility or sagittal plane kinematics between the two visits were analyzed. In addition, correlations were performed to analyze any potential relationships between the variables of interest.

While the results found an increase in passive hip extension following the stretching program, no significant changes in the sagittal plane kinematics were found. In addition, no relationships between the change in passive hip extension and the change in either peak active hip extension, peak anterior pelvic tilt, or peak lumbar spine extension were 
discovered. The lack of relationship between the change in passive hip extension and the change in active hip extension suggest that flexibility is not the only factor that influences range of motion at the hip during dynamic movements. Other potential factors influencing the hip range of motion include the geometric parameters of the joint and the speed that the movement is performed at.

Although passive hip extension was not related to active hip extension, anterior pelvic tilt, or lumbar spine extension, several relationships were noted. Correlations observed between hip extension, anterior pelvic tilt, and lumbar spine extension during running show that a relationship does exist between the movements of these three segments. That relationship is important to understand, as it may provide insight into an underlying mechanism that helps to explain the cause of low back pain in runners.

\section{Conclusion}

The results of the present study demonstrated that a three-week static stretching program of the hip flexor musculature resulted in an increase in passive hip extension, but no change in the sagittal plane kinematics of the hips, pelvis, and spine during running. The absence of any changes in the kinematics of the LPH complex, as well as the absence of a correlation between passive and active hip extension, suggest that muscular flexibility is not the only factor that determines range of motion at the hip during running at submaximal speeds. However, the correlations observed between hip extension, anterior pelvic tilt, and lumbar spine extension during running suggest a kinematic relationship exists between the segments making up the LPH complex. This relationship should be 
explored further in runners with LBP, as the sagittal plane kinematics of the LPH complex may offer a theoretical mechanism to help explain pain in this population.

\section{Recommendations for Future Research}

In the future, it would be helpful to analyze the effects of a stretching program on the sagittal plane kinematics during running at a faster speed. The current study analyzed the running kinematics at a submaximal speed, which may have limited the results we saw. Additionally, collecting oxygen uptake as a measure of running economy may help to provide additional information regarding the influence of passive hip extension flexibility on the sagittal plane kinematics of the LPH complex during running. Finally, the same study could be conducted on a low back pain population of runners. Doing so would allow comparisons to be made between a healthy population and a low back pain population,

potentially giving insight into an underlying mechanism that would help to explain one of the causes of low back pain in runners. 


\section{Appendix A: Marker Set}
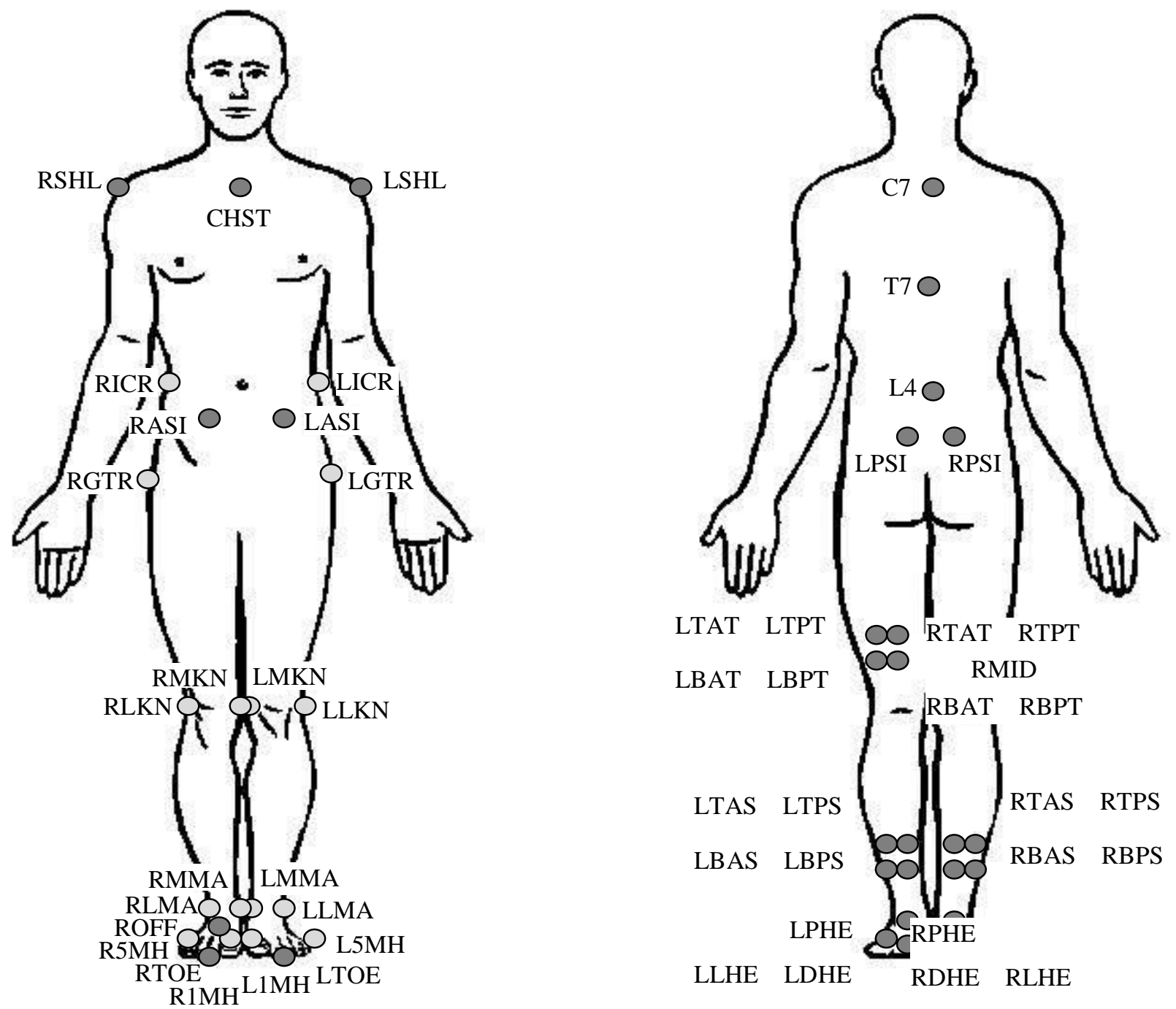

$\bigcirc$ Tracking Marker
$\bigcirc$ Anatomical Marker 


\section{Appendix B: Model Definition}

\section{Pelvis:}

A CODA pelvis was used to define the pelvic coordinate system. A plane was defined using LASI and RASI and the midpoint of the LPSI and RPSI (Appendix A). The mediolateral (X) axis was defined from the midpoint of the LASI and RASI towards RASI. The vertical (Z) axis was perpendicular to the plane formed by the ASIS and PSIS markers. The antero-posterior (Y) axis was defined as the cross-product of the $\mathrm{X}$-axis and Z-axis with its positive direction anterior.

\section{Thigh:}

A plane was defined using the hip joint center (35) and the RMKN and RLKN markers. The vertical axis (Z) was defined as the vector originating at the midpoint of RMKN and RLKN with its positive direction pointing towards the hip joint center. The antero-posterior axis (Y) was perpendicular to the plane formed by RMKN and RLKN and the hip joint center with its positive direction anterior. Lastly, the cross product of the first two axes defined the medio-lateral axis $(\mathrm{X})$ with its positive direction to the right. The tracking markers were a rigid cluster of four (LTAT, LTPT, LBAT, and LBPT) or five markers (RTAT, RTPT, RBAT, RBPT, and RMID) placed on the lateral and posterior side of the distal thigh.

\section{Shank:}

A plane was defined using RMKN and RLKN and RMMA and RLMA. The vertical axis (Z) originated at the midpoint of RMMA and RLMA with its positive direction pointing to 
the midpoint of RMKN and RLKN. The antero-posterior axis $(\mathrm{Y})$ was perpendicular to the plane formed by RMKN, RLKN, RMMA, and RLMA with the anterior direction positive. The cross-product of the $\mathrm{Y}$ and $\mathrm{Z}$ axes gave the medio-lateral axis $(\mathrm{X})$ with its positive direction to the right. The tracking markers were a rigid cluster of four markers (RTAS, RTPS, RBAS, and RBPS) placed on the lateral and posterior side of the distal shank.

\section{Foot:}

The vertical ( $\mathrm{Z}$ ) axis was defined as the vector originating at RDHE with the positive direction pointing to the RPHE. The antero-posterior axis $(\mathrm{Y})$ was parallel to the floor and was defined as the vector from RDHE to the midpoint of R1MH and R5MH with the anterior direction positive. The cross-product of the $\mathrm{Y}$ and $\mathrm{Z}$ axes gave the medio-lateral axis (X) with the positive direction to the right. Tracking markers included RDHE, RPHE, and RLHE.

\section{Trunk:}

A plane was defined using RSHL, LSHL, RICR, and LICR. The vertical axis (Z) originated at the midpoint of RICR and LICR with its positive direction pointing towards the midpoint of RSHL and LSHL. The antero-posterior axis (Y) was perpendicular to the plane formed by RSHL, LSHL, RICR, and LICR with the anterior direction positive. The cross product of the $\mathrm{Y}$ and $\mathrm{Z}$ axes gave the medio-lateral axis $(\mathrm{X})$ with its positive direction to the right. Tracking markers included CHST, C7, T7, and L4. 


\section{Appendix C: Expanded Literature Review}

\section{Running Injuries}

Running is one of the most popular recreational activities in the United States, with over 50 million people participating each year in the United States alone (1). Unfortunately, participation in running is often accompanied by injury. Running injuries are common, with annual incidence rates ranging from between $19.4 \%$ to $92.4 \%$, depending on the specific definition of a "running injury" (2). Generally, it is accepted that approximately 50\% of runners experience an injury each year (3). According to Lopes et al. (4), low back pain accounts for approximately 5.5\% of those injuries.

\section{Mechanisms of Low Back Pain}

Low back pain is often differentiated into three categories: specific spinal pathology, nerve root pain, and simple or non-specific low back pain (36). Low back pain is a common ailment; it has been estimated that up to $84 \%$ of the general population have dealt with low back pain at some point in their lives (37), with non-specific low back pain accounting for approximately $85 \%$ of low back pain patients seen in a primary care setting (38). Despite the high prevalence rate, the underlying mechanisms that cause this condition are poorly understood.

Much of the literature related to non-specific low back pain has focused on either muscular dysfunction of the trunk/spine region (39-41) or the muscles surrounding the hips (42-44) as potential contributors to low back pain. However, there are also studies that focus on the kinematics of the trunk, pelvis, and lower extremities as a way to help explain 
low back pain (30-34). To explain the how the interaction between the muscular dysfunction of the trunk and hips and abnormal kinematics of the hip, pelvis, and lumbar spine could potentially contribute to low back pain, Janda (45-47) proposed the concept of the Pelvic Crossed Syndrome. The Pelvic Crossed Syndrome is characterized by hyperactivity and tightness at the hip flexors and spinal erectors, and weakness and increased length of the gluteal and abdominal muscles. These muscular imbalances are typically accompanied by anterior tilt of the pelvis, increased flexion of the hips, and hyperlordosis of the lumbar spine, which can overstress the hip joints and lower back. The Pelvic Crossed Syndrome has been found in patients dealing with non-specific low back pain, and therefore may help to explain the underlying mechanisms for low back pain.

\section{Anatomy of Lumbo-Pelvic-Hip Complex}

The Pelvic Crossed Syndrome was proposed as a way to explain the muscular and kinematic interactions between the lumbar spine, pelvis, and hips. The lumbo-pelvic-hip (LPH) complex consists of the five vertebrae of the lumbar spine, the pelvic girdle, and the hip joint (48).

\section{Lumbar Spine}

The spinal column is made up of 24 articulating vertebrae and nine that are fused. The column can be further divided into five sections. The most superior seven vertebrae are known as the cervical vertebrae. They are followed by the 12 thoracic vertebrae and the five lumbar vertebrae. The most inferior sections of the vertebral column are the sacrum and coccyx, which are made up of five and four fused vertebrae, respectively. Except for the atlantoaxial joint, which is made up of the first two cervical vertebrae, the intervertebral 
joints only allow for limited movement between any two vertebrae. It is the cumulative effect of the movements of each intervertebral joint that allows for substantial movement of the spine in any direction. The rest of the vertebral articulations are classified as arthrodial joints because of the limited gliding movement that can occur between the joints. In between each vertebrae are the intervertebral disks, which is made up of elastic material. The placement of the intervertebral disks allows for compression in all directions (49).

The movable vertebrae of the spine have three normal curves. The cervical and lumbar sections of the spine naturally have an anterior convex curve to them, while the thoracic section has an anterior concave curve to it. The normal curves of the spine better enable it to absorb blows and shocks. However, an excessive posterior concave curve of the cervical or lumbar section, known as lordosis, can occur. Excessive anterior concavity of the thoracic section, known as kyphosis, can also occur (49). Lordosis or kyphosis may cause repetitive impingement of the vertebral facets due to excessive forces being placed on a more localized area of the intervertebral disk, which is thought to be related to the onset of low back pain (25).

\section{Pelvic Girdle and Hip Joint}

The pelvic girdle consists of the right and left pelvic bone joined together by the sacrum of the spinal column. Each of the pelvic bones are made up of three areas: the ilium, the ischium, and the pubis. The ilium makes up the upper two fifths of the pelvic bone, while the posterior lower two fifths is the ischium and the anterior lower one fifth is the pubis. The lateral aspect of the pelvic bone includes the acetabulum, which is a cup-shaped structure into which the femoral head fits. The hip joint, also known as the acetabular 
femoral joint, is a ball and socket joint. The structure of the ball and socket joint allows for a wide range of motion at the hip in all three planes of motion (49).

\section{Muscles of the Hip Joint}

There are 21 muscles that contribute to motion at the hip joint in all three planes. In the sagittal plane, the muscles utilized primarily to produce hip flexion movement are the iliopsoas, sartorius, tensor fasciae latae, rectus femoris, adductor longus, and pectineus, while the muscles that produce hip extension movement are the gluteus maximus, adductor magnus, biceps femoris, semitendinosus, and semimembranosus. In the frontal plane, the pectineus, gracilis, adductor longus, adductor brevis, and adductor magnus all produce adduction, while the gluteus medius, gluteus minimus, and tensor fasciae latae produce abduction. The transverse plane external rotators are the gluteus maximus, piriformis, gemellus superior, gemellus inferior, and quadratus femoris. The internal rotators include the gluteus minimus, gluteus medius, tensor fasciae latae, adductor longus, adductor brevis, adductor magnus, and pectineus (50).

The hyperactivity and tightness of the hip flexor muscles found in individuals who exhibit symptoms of the Pelvic Crossed Syndrome relate this muscle group to LBP. Of the six hip flexor muscles, the iliopsoas, tensor fasciae latae, adductor longus, and pectineus muscles are all one-joint muscles, meaning they only cross the hip joint. On the other hand, the rectus femoris and sartorius muscles are two-joint muscles, which means that they cross both the hip and knee joints (49).

The iliopsoas is often divided into two separate parts: the iliacus and the psoas. The origin of the iliacus is on the inner surface of the ilium, while it inserts into the lesser 
trochanter of the femur. The psoas muscle originates on the lumbar vertebrae, the sides of the bodies of the last thoracic vertebrae, the intervertebral fibrocartilages, and the base of the sacrum. The psoas major also inserts into the lesser trochanter of the femur, similar to the iliacus, while the psoas minor inserts in the pectineal line of the pubis, which is located on the front of the pubis just above the crest, and the iliopectineal eminence. The tensor fasciae latae originates on the anterior iliac crest and the surface of the ilium, while it inserts a quarter of the way down the thigh into the iliotibial tract. The origin of the adductor longus is anterior pubis, while its insertion is in the middle third of the linea aspera. The pectineus is the last one-joint muscle that produces hip flexion. It originates on the pectineal line and inserts into the pectineal line of the femur, which is located below the lesser trochanter towards the linea aspera (49).

The two-joint muscles, which include the rectus femoris and the sartorius, originate on the pelvis but cross both the hip and knee joints to insert on the tibia. The rectus femoris originates at the anterior interior iliac spine of the ilium and the groove above the acetabulum. It inserts at both the superior aspect of the patella and the patellar tendon to the tibial tuberosity. The sartorius originates at the anterior superior iliac spine and the notch just below the spine, while it inserts on the anterior medial surface of the tibia just below the condyle (49).

\section{Kinematics of Lumbo-Pelvic-Hip Complex and Low Back Pain}

Normal biomechanics of gait consist of complex coordinated movement patterns of the hip, pelvis, and lumbar spine $(7,9,22,48,51)$. Deviations from normal gait kinematics can potentially place an individual at risk for injury due to altered loading patterns on the 
spine and lower extremity joints. Therefore, it is possible that abnormal kinematics play a role in low back pain.

Hip Extension and Anterior Pelvic Tilt

Although the exact underlying cause of non-specific low back pain (LBP) in runners is currently unknown, research suggests the existence of a kinematic relationship between the hips, pelvis, and lumbar spine. Limited "passive” hip extension flexibility, defined as the range of motion measured in a static position, is thought to cause an increase in both anterior pelvic tilt and lumbar lordosis during running (10). In 14 healthy track and field athletes, Schache et al. (7) found a significant positive correlation between anterior pelvic tilt and “active” peak hip extension flexibility during a dynamic activity, which in this case was running. Anterior pelvic tilt tended to be greater in runners who displayed reduced active peak hip extension during terminal stance of running. Similar results were found by Franz et al. (8), who reported a significant positive correlation between peak anterior pelvic tilt and active peak hip extension during both walking $(r=0.90, \mathrm{p}<0.01)$ and running $(r=0.83, p, 0.01)$ in a group of 75 healthy, recreational runners. Kerrigan et al. (52) reported the same positive correlation ( $r=0.69$, $\mathrm{p}<0.01$ ) in a healthy elderly population, suggesting that this relationship exists across different populations.

\section{Anterior Pelvic Tilt and Lumbar Spine Extension}

In addition to the correlation between limited active hip extension flexibility and greater anterior pelvic tilt, a relationship has also been established between anterior pelvic

tilt and extension of the lumbar spine. This relationship has been shown to exist during static standing (53), walking (54), and running (9). In a sample made up of 20 injury-free 
male runners, Schache et al. (9) reported a high correlation between anterior tilt of the pelvis and extension of the lumbar spine $(r=-0.84)$. The correlations between hip extension and anterior pelvic tilt, as well as between anterior pelvic tilt and lumbar spine extension, suggest a kinematic relationship between the segments of the lumbo-pelvic-hip (LPH) complex during running. However, to our knowledge, no relationship has been established between active hip extension and lumbar spine extension during running.

\section{Flexibility in Research}

Passive vs. Active Flexibility

Flexibility of the hip flexors can be defined in terms of passive flexibility or active flexibility. Passive flexibility is measured during a static position, such as during the modified Thomas test (MTT), while active flexibility is measured during a dynamic movement, such as running.

Modified Thomas Test

The MTT is a test used to measure passive hip extension flexibility. In the test, participants are positioned on their back with their measured hip at the edge of the treatment table and their tested leg hanging passively off the table. At the same time, the opposite leg is held by the participant with the hip and knee in a flexed position against the chest. Participants are instructed to pull their knee straight to their head in order to prevent any hip abduction. Care is also be taken to ensure that the participant's low back lays flat against the table so that the pelvis and lumbar spine remain in a neutral position throughout the entire test. Using a digital inclinometer placed on the thigh, the hip extension flexibility will be measured. According to Kendall et al. (55), the angle that the thigh produces relative 
to the horizontal defines the flexibility of the hip flexor muscles, including the iliacus, psoas, pectineus, rectus femoris, and sartorius. Individuals are considered to have sufficient flexibility if the thigh drops below the horizontal, but reduced passive hip extension flexibility if the thigh remains above the horizontal.

The accuracy and reliability of the MTT may be affected by variations in the assessment skill of the investigator, consistency of the assessment procedure, accuracy of the measurement equipment, or the scoring method (28). While its use as an assessment technique for hip extension flexibility is well established, the reliability of the MTT has been questioned (28). However, other studies have demonstrated the excellent test-retest reliability of the MTT (ICC $=0.97, \mathrm{SEM}=1.51, \mathrm{MDD}=4.17)(16)$. Similar results were found by Cejudo et al. (56), who reported intraclass correlation coefficients of at least 0.87 on three different testing sessions of the MTT.

\section{Gender and Flexibility}

Research shows that females are more flexible than males at most joints in the body (57-61). However, it is unclear whether males and females produce similar improvements in flexibility in response to a stretching program. Cipriani et al. (57) found that both males and females demonstrated similar improvements in flexibility when participating in a fourweek hamstring stretching program. While females displayed greater hip range of motion than males both at baseline and after the four weeks of stretching, the rate of change over time was not significantly different between genders. However, these findings contradict the results of Starring et al. (61), who reported that females saw greater improvements in 
hamstring flexibility following five days of static or cyclic stretching than their male counterparts.

\section{Hip Extension and Low Back Pain}

\section{Normal Population}

The kinematic correlations between the segments of the LPH complex during running suggests a relationship between hip extension flexibility and LBP. Evidence to support the relationship shows that individuals with non-specific LBP display significantly less passive hip extension than healthy individuals (6). Similar results were reported by Roach et al. (5), who discovered that passive hip extension flexibility was significantly greater in the active healthy group $\left(6.78 \pm 7.18^{\circ}\right)$ compared to the active LBP group (-4.16 $\pm 8.81^{\circ}$ ). While the studies by Van Dillen et al. (6) and Roach et al. (5) were not conducted on a running population, the kinematic behavior of the LPH complex may provide insight into the underlying cause of low back pain during running.

\section{Running Population}

While limited passive hip extension has been associated with non-specific LBP in a normal population, causation for the reduced range of motion during running has yet to be established. It is possible that reduced passive flexibility of the hip flexor muscle group may contribute to the reduction in active hip extension during running. In an attempt to explain a potential cause of LBP in runners, Wang et al. (62) compared the passive hip extension flexibility between a running and non-running group using the MTT. While they discovered no difference in flexibility between the groups, the aforementioned authors suggested that their results may have been due to many of their participants from both the 
running and non-running groups spending a significant portion of their day in a seated position. This would place the hip flexor muscles in a shortened range and may influence the extensibility of these muscles. On the other hand, a study conducted by Bach et al. (10) compared hip flexibility between runners and non-runners. The researchers found reduced passive flexibility of the hip flexors in the running group. Furthermore, the researchers separated the runners into a back pain group and a healthy group to explore the relationship between LBP and hip flexor tightness. While they did not find a relationship between reduced passive hip extension flexibility and LBP, their small sample size $(\mathrm{N}=10)$ limited the conclusions of their study, and thus further investigation into the relationship between hip flexor flexibility and LBP is warranted.

\section{Improving Flexibility to Reduce the Symptoms of Low Back Pain}

\section{Frequency and Duration of Static Stretching}

Static stretching is a method to increase muscular extensibility in which the muscle is slowly lengthened to tolerance and then held at that position for a period of time. Numerous studies have attempted to identify the optimal frequency and duration that static stretching must be performed to gain improvements in flexibility (63-70). It has been shown that during a single bout of static stretching, a 15 second hold is just as effective at increasing hip abduction flexibility as a 45 or 120 second hold (63). However, when a six week stretching program was performed, results showed that a single repetition of either a 30 second or 60 second hold was more effective at improving flexibility than a single repetition of a 15 second hold (64). In addition, there was no significant difference in flexibility improvements between the 30 second hold or the 60 second hold. These results 
suggest that to see prolonged improvements in flexibility, the duration of the static stretch must be at least 30 seconds.

Other studies have since shown that the duration of the stretch hold position may not be as important as the total amount of time spent stretching per day. For example, Roberts and Wilson (66) reported that after five weeks of static stretching of the hamstrings, nine repetitions with a five second hold produced the same flexibility improvements as three repetitions with a 15 second hold. Similar results have been found by other studies $(65,67-70)$, thus indicating that as long as the total amount of time spent in a stretch hold is equal, similar improvements in flexibility will be observed.

While the frequency and duration of a stretch are important factors to consider, the duration of the stretching program must be considered as well to see improvements in flexibility. While many research studies that implement a static stretching program last at least five weeks (64-69, 71), Winters et al. (11) found that three weeks of static stretching was sufficient to significantly increase the passive flexibility of the hip flexors. The study measured flexibility at baseline, three weeks, and six weeks, and defined a positive measurement as one in which the thigh dropped below the horizontal. The passive flexibility of the hip flexors increased significantly from baseline to three weeks (from -11 $\pm 4^{\circ}$ to $2 \pm 5^{\circ}$ ), but there was no significant difference between the measurements at three weeks and six weeks ( $2 \pm 5^{\circ}$ and $3 \pm 4^{\circ}$, respectively).

\section{Static Stretching of the Hip Flexors}

Based on the relationships reported between reduced passive hip extension flexibility and non-specific LBP in a normal population $(5,6)$, improving flexibility of the 
hip flexors may be a useful intervention to reduce LBP. In fact, Avrahami and Potvin (72) found that five sessions of manual fascial-muscular lengthening therapy of the iliopsoas complex was effective at increasing flexibility and decreasing pain in a population of low back pain patients.

The improvements in passive hip extension flexibility reported by Avrahami and Potvin (72) were seen in individuals with LBP; similar results have also been found in healthy populations. In individuals with limited passive hip extension flexibility, improvements have been found following both a single session of static stretching (73) and after a six week static stretching program (11). Moreside and McGill (12) reported similar improvements in passive hip extension flexibility when they implemented a six week passive stretching program in six healthy male individuals with limited passive hip extension flexibility. Participants performed either a modified lunge stretch or a supine leg extension stretch for 30 seconds at least four days a week. Following the six weeks, the participants exhibited an average increase in the passive hip extension flexibility of $14^{\circ}$ (pretreatment $=7.8 \pm 3^{\circ}$, posttreatment $=-5.8 \pm 6^{\circ}$ ).

Although increases in passive hip extension flexibility following a static stretching program have been found, similar improvements in active hip extension during running following a static stretching program have not been documented. Moreside and McGill (13) investigated the effects of increased passive hip extension flexibility on active hip extension flexibility during several dynamic movements. In the study, 24 healthy male participants with limited passive hip extension flexibility performed four dynamic movements to measure active hip extension flexibility following six weeks of passive hip extension stretching. The four movements included an active hip extension in upright 
standing, a lunge, a standing “twist and reach" maneuver, and exercising on an elliptical trainer. Despite the significant increase in passive hip extension flexibility, there were no significant changes in active hip extension flexibility during the dynamic movement tasks. However, the dynamic movement tasks performed may not be representative of the movements of the LPH complex during running. No study has yet examined the effects of static hip flexor stretching on active hip extension flexibility during running.

\section{Summary}

In conclusion, it is important to study the influence of a static hip flexor stretching program on active hip extension flexibility during running. Understanding the relationship between passive and active hip extension flexibility may bring insight into the interactions between kinematics of the LPH complex during running. Abnormal kinematics of the LPH complex are one aspect of the Pelvic Crossed Syndrome, which has been found in nonspecific LBP patients. Therefore, the findings of such a study may have implications for runners suffering from non-specific LBP. 


\section{REFERENCES}

1. State of the Sport - Part II: Running Industry Report [Web Site]. 2014 [cited 2015 August 15]. Available from: http://www.runningusa.org/2014-running-industryreport?returnTo=annual-reports.

2. van Gent RN, Siem D, van Middelkoop M, van Os AG, Bierma-Zeinstra SM, Koes BW. Incidence and determinants of lower extremity running injuries in long distance runners: a systematic review. Br J Sports Med. 2007;41(8):469-80; discussion 80.

3. Fields KB, Sykes JC, Walker KM, Jackson JC. Prevention of running injuries. Current Sports Medicine Reports. 2010;9(3):176-82.

4. Lopes AD, Hespandhol JLC, Yeung SS, Costa LO. What are the main running-related musculoskeletal injuries? A Systematic Review. Sports Medicine. 2012;42(10):891-905.

5. Roach SM, San Juan JG, Suprak DN, Lyda M, Bies AJ, Boydston CR. Passive hip range of motion is reduced in active subjects with chronic low back pain compared to controls. International Journal of Sports Physical Therapy. 2015;10(1):13-20.

6. Van Dillen LR, McDonnell MK, Fleming DA, Sahrmann SA. Effect of knee and hip position on hip extension range of motion in individuals with and without low back pain. Journal of Orthopaedic \& Sports Physical Therapy. 2000;30(6):307-16.

7. Schache AG, Blanch PD, Murphy AT. Relation of anterior pelvic tilt during running to clinical and kinematic measures of hip extension. Br J Sports Med. 2000;34(4):279-83.

8. Franz JR, Paylo KW, Dicharry J, Riley PO, Kerrigan DC. Changes in the coordination of hip and pelvis kinematics with mode of locomotion. Gait Posture. 2009;29(3):494-8.

9. Schache AG, Blanch PD, Rath D, Wrigley T, Bennell K. Three-dimensional angular kinematics of the lumbar spine and pelvis during running. Human Movement Science. 2002;21(2):273-93.

10. Bach DK, Green DS, Jensen GM, Savinar E. A comparison of muscular tightness in runners and nonrunners and the relation of muscular tightness to low back pain in runners. The Journal of Orthopaedic and Sports Physical Therapy. 1985;6(6):315-23.

11. Winters MV, Blake CG, Trost JS, Marcello-Brinker TB, Lowe L, Garber MB, et al. Passive versus active stretching of hip flexor muscles in subjects with limited hip extension: $A$ randomized clinical trial. Phys Ther. 2004;84(9):800-7.

12. Moreside JM, McGill SM. Hip joint range of motion improvements using three different interventions. Journal of Strength and Conditioning Research. 2012;26(5):1265-73.

13. Moreside JM, McGill SM. Improvements in hip flexibility do not transfer to mobility in functional movement patterns. Journal of Strength and Conditioning Research. 2013;27(10):2635-43.

14. Watt JR, Jackson K, Franz JR, Dicharry J, Evans J, Kerrigan DC. Effect of a supervised hip flexor stretching program on gait in elderly individuals. PM R. 2011;3(4):324-9.

15. Moreside JM, McGill SM. Quantifying normal 3D hip ROM in healthy young adult males with clinical and laboratory tools: hip mobility restrictions appear to be plane-specific. Clin Biomech (Bristol, Avon). 2011;26(8):824-9.

16. Kim G, Ha S. Reliability of the modified Thomas test using a lumbo-pelvic stabilization. J Phys Ther Sci. 2015;27(2):447-9.

17. Zeni JA, Jr., Higginson JS. Gait parameters and stride-to-stride variability during familiarization to walking on a split-belt treadmill. Clin Biomech (Bristol, Avon). 2010;25(4):3836.

18. Junqueira LD, Amaral LQ, lutaka AS, Duarte M. Effects of transporting an infant on the posture of women during walking and standing still. Gait Posture. 2015;41(3):841-6. 
19. Hinkle DE, Wiersma W, Jurs SG. Applied statistics for the behavioral sciences. 5th ed. Boston: Houghton Mifflin; 2003. xxi, 756 p. p.

20. Taylor DC, Dalton JD, Seaber AV, Garrett WE. Viscoelastic properties of muscle-tendon units. The American Journal of Sports Medicine. 1990;18(3):300-9.

21. Wilson DR, Feikes JD, O'Connor JJ. Ligaments and articular contact guide passive knee flexion. J Biomech. 1998;31(12):1127-36.

22. Whittle MW, Levine D. Three-dimensional relationships between the movements of the pelvis and lumbar spine during normal gait. Human Movement Science. 1999;18:681-92.

23. Stanish W. Low back pain in middle-aged athletes. Am J Sports Med. 1979;7(6):367-9.

24. Slocum DB, James SL. Biomechanics of running. Jama. 1968;205(11):721-8.

25. Schache AG, Blanch PD, Rath DA, Wrigley TV, Bennell KL. Are anthropometric and kinematic parameters of the lumbo-pelvic-hip complex related to running injuries? Res Sports Med. 2005;13(2):127-47.

26. Klein KK, Roberts CA. Mechanical problems of marathoners and joggers. Cause and solution. American corrective therapy journal. 1976;30(6):187-91.

27. Geraci MC, Jr. Overuse injuries of the hip and pelvis. Journal of back and musculoskeletal rehabilitation. 1996;6(1):5-19.

28. Peeler J, Anderson JE. Reliability of the Thomas test for assessing range of motion about the hip. Physical Therapy in Sport. 2007;8(1):14-21.

29. Stewart IB, Sleivert GG. The effect of warm-up intensity on range of motion and anaerobic performance. J Orthop Sports Phys Ther. 1998;27(2):154-61.

30. Hamill J, Moses M, Seay J. Lower extremity joint stiffness in runners with low back pain. Res Sports Med. 2009;17(4):260-73.

31. Seay JF, Van Emmerik RE, Hamill J. Low back pain status affects pelvis-trunk coordination and variability during walking and running. Clin Biomech (Bristol, Avon). 2011;26(6):572-8.

32. Muller R, Ertelt T, Blickhan R. Low back pain affects trunk as well as lower limb movements during walking and running. J Biomech. 2015;48(6):1009-14.

33. Lamoth CJ, Meijer OG, Wuisman PI, van Dieen JH, Levin MF, Beek PJ. Pelvis-thorax coordination in the transverse plane during walking in persons with nonspecific low back pain. Spine (Phila Pa 1976). 2002;27(4):E92-9.

34. Youdas JW, Garrett TR, Egan KS, Therneau TM. Lumbar lordosis and pelvic inclination in adults with chronic low back pain. Phys Ther. 2000;80(3):261-75.

35. Bell AL, Brand RA, Pedersen DR. Prediction of hip joint centre location from external landmarks. Human Movement Science. 1989;8(1):3-16.

36. Waddell G. The back pain revolution. 2nd ed. Edinburgh ; New York: Churchill Livingstone; 2004. xiii, 475 p. p.

37. Walker BF. The prevalence of low back pain: a systematic review of the literature from 1966 to 1998. Journal of spinal disorders. 2000;13(3):205-17.

38. Deyo RA, Phillips WR. Low back pain. A primary care challenge. Spine (Phila Pa 1976). 1996;21(24):2826-32.

39. D'Hooge R, Hodges P, Tsao H, Hall L, Macdonald D, Danneels L. Altered trunk muscle coordination during rapid trunk flexion in people in remission of recurrent low back pain. Journal of electromyography and kinesiology : official journal of the International Society of Electrophysiological Kinesiology. 2013;23(1):173-81.

40. MacDonald D, Moseley GL, Hodges PW. People with recurrent low back pain respond differently to trunk loading despite remission from symptoms. Spine (Phila Pa 1976). 2010;35(7):818-24. 
41. Hosseinifar M, Akbari M, Behtash H, Amiri M, Sarrafzadeh J. The Effects of Stabilization and Mckenzie Exercises on Transverse Abdominis and Multifidus Muscle Thickness, Pain, and Disability: A Randomized Controlled Trial in NonSpecific Chronic Low Back Pain. J Phys Ther Sci. 2013;25(12):1541-5.

42. Kendall KD, Schmidt C, Ferber R. The relationship between hip-abductor strength and the magnitude of pelvic drop in patients with low back pain. J Sport Rehabil. 2010;19(4):422-35. 43. Mok NW, Brauer SG, Hodges PW. Hip strategy for balance control in quiet standing is reduced in people with low back pain. Spine (Phila Pa 1976). 2004;29(6):E107-12.

44. Kankaanpaa M, Taimela S, Laaksonen D, Hanninen O, Airaksinen O. Back and hip extensor fatigability in chronic low back pain patients and controls. Arch Phys Med Rehabil. 1998;79(4):412-7.

45. Janda V, Frank C, Liebenson C. Evaluation of Muscular Imbalance. In: Liebenson C, editor. Rehabilitation of the Spine: A practitioner's Manual. second ed. Philadelphia: Lippincott Williams \& Wilkins; 2007.

46. Janda $\mathrm{V}$, Schmid $\mathrm{H}$. Muscles as a pathogenic factor in back pain. The treatment of patients. 1980:1-21.

47. Jull G, Janda V. Muscles and motor control in low back pain: assessment and management. Physical therapy of the low back New York: Churchill Livingstone. 1987:253-78. 48. Schache AG, Bennell KL, Blanch PD, Wrigley TV. The coordinated movement of the lumbo-pelvic-hip complex during running: a literature review. Gait Posture. 1999;10(1):30-47. 49. Floyd RT. Manual of structural kinesiology. 18th ed. New York, NY: McGraw-Hill; 2012. vii, 408 p. p.

50. Neumann DA. Kinesiology of the hip: a focus on muscular actions. J Orthop Sports Phys Ther. 2010;40(2):82-94.

51. Saunders SW, Schache A, Rath D, Hodges PW. Changes in three dimensional lumbopelvic kinematics and trunk muscle activity with speed and mode of locomotion. Clin Biomech (Bristol, Avon). 2005;20(8):784-93.

52. Kerrigan DC, Lee LW, Collins JJ, Riley PO, Lipsitz LA. Reduced hip extension during walking: healthy elderly and fallers versus young adults. Arch Phys Med Rehabil. 2001;82(1):2630.

53. Levine $D$, Whittle MW. The effects of pelvic movement on lumbar lordosis in the standing position. J Orthop Sports Phys Ther. 1996;24(3):130-5.

54. Levine $D$, Richards J, Whittle M, Whittle M. Whittle's gait analysis. 5th ed. Edinburgh ; New York: Churchill Livingstone/Elsevier; 2012. xi, 177 p. p.

55. Kendall FP, McCreary EK, Provance PG. Muscles, testing and function : with Posture and pain. 4th ed. Baltimore, Md.: Williams \& Wilkins; 1993. xv, 451 p. p.

56. Cejudo A, Sainz de Baranda P, Ayala F, Santonja F. Test-retest reliability of seven common clinical tests for assessing lower extremity muscle flexibility in futsal and handball players. Phys Ther Sport. 2015;16(2):107-13.

57. Cipriani DJ, Terry ME, Haines MA, Tabibnia AP. Effect of stretch frequency and sex on the rate of gain and rate of loss in muscle flexibility during a hamstring-stretching program: a randomized single-blind longitudinal study. Journal of Strength and Conditioning Research. 2012;26(8):2119-29.

58. Alter MJ, Alter MJ. Science of flexibility. 2nd ed. Champaign, IL: Human Kinetics; 1996. xvii, 373 p. p.

59. Allander E, Bjornsson OJ, Olafsson O, Sigfusson N, Thorsteinsson J. Normal range of joint movements in shoulder, hip, wrist and thumb with special reference to side: a comparison between two populations. International journal of epidemiology. 1974;3(3):253-61. 
60. Bell RD, Hoshizaki TB. Relationships of age and sex with range of motion of seventeen joint actions in humans. Canadian journal of applied sport sciences Journal canadien des sciences appliquees au sport. 1981;6(4):202-6.

61. Starring DT, Gossman MR, Nicholson Jr. GG, Lemons J. Comparison of cyclic and sustained passive stretching using a mechanical device to increase resting length of hamstring muscles. Phys Ther. 1988;68(3):314-20.

62. Wang SS, Whitney SL, Burdett RG, Janosky JE. Lower extremity muscular flexibility in long distance runners. J Orthop Sports Phys Ther. 1993;17(2):102-7.

63. Madding SW, Wong JG, Hallum A, Medeiros J. Effect of duration of passive stretch on hip abduction range of motion. J Orthop Sports Phys Ther. 1987;8(8):409-16.

64. Bandy WD, Irion JM. The effect of time on static stretch on the flexibility of the hamstring muscles. Physical Therapy in Sport. 1994;74(9):845-52.

65. Bandy WD, Irion JM, Briggler M. The effect of time and frequency of static stretching on flexibility of the hamstring muscles. Phys Ther. 1997;77(10):1090-6.

66. Roberts JM, Wilson $\mathrm{K}$. Effect of stretching duration on active and passive range of motion in the lower extremity. Br J Sports Med. 1999;33(4):259-63.

67. Cipriani DJ, Abel B, Pirrwitz D. A comparison of two stretching protocols on hip range of motion: Implications for total daily stretch duration. Journal of Strength and Conditioning Research. 2003;17(2):274-8.

68. Ayala F, Sainz de Baranda Andujar P. Effect of 3 Different Active Stretch Durations on hip flexion range of motion. Journal of Strength and Conditioning Research. 2010;24(2):430-6.

69. Johnson AW, Mitchell UH, Meek K, Feland JB. Hamstring flexibility increases the same with 3 or 9 repetitions of stretching held for a total time of 90 s. Phys Ther Sport. 2014;15(2):101-5.

70. Ford GS, Mazzone MA, Taylor K. The Effect of 4 Different Durations of Static Hamstring Stretching on Passive Knee-Extension Range of Motion. J Sport Rehabil. 2005;14:95-107.

71. Feland JB, Myrer JW, Schulthies SS, Fellingham GW, Measom GW. The effect of duration of stretching of the hamstring muscle group for increasing range of motion in people aged 65 years or older. Phys Ther. 2001;81(5):1110-7.

72. Avrahami D, Potvin JR. The clinical and biomechanical effects of fascial-muscle lengthening therapy on tight hip flexor patients with and without low back pain. J Can Chiropr Assoc. 2014;58(4):444-55.

73. Godges JJ, MacRae H, Longdon C, Tinberg C, MacRae P. The effects of two stretching procedures on hip range of motion and gait economy. The Journal of Orthopaedic and Sports Physical Therapy. 1989;10(9):350-7. 
Author's Name: Jeffrey Howard Mettler

Place of Birth: Sioux Falls, South Dakota, United States

$\underline{\text { Education }}$

Bachelor of Science in Kinesiology \& Sport Science

University of South Dakota

May 2014

$\underline{\text { Professional Positions }}$

Teaching Assistant, University of Kentucky, August 2014 - May 2016

$\underline{\text { Scholastic Honors }}$

School of Education Outstanding Leadership Award, University of South Dakota, 2014 Carl \& Hazel Hoy Award, University of South Dakota, 2014

Summit League Scholar-Athlete of the Year, 2013 